\title{
1 Dopamine neuron synaptic connectivity defines physiological striatal domains
}

\author{
Nao Chuhma*, Soo Jung Oh, Stephen Rayport*
}

3

4 Department of Molecular Therapeutics, New York State Psychiatric Institute, New York, NY

$5 \quad 10032$, USA

6 Department of Psychiatry, Columbia University, New York, NY 10032, USA

$7 \quad *$ Correspondence to: nao.chuma@nyspi.columbia.edu, stephen.rayport@nyspi.columbia.edu

9 Abstract

10 Dopamine neurons projecting to the striatum control movement, cognition, and motivation. They

11 do so via slower, dopamine volume transmission and also via faster synaptic dopamine,

12 glutamate and GABA transmission. To define the scope of these synaptic actions, we recorded

13 dopamine neuron synaptic currents in the four major classes of striatal neurons. This revealed

14 that dopaminergic and GABAergic synaptic actions are widespread; glutamatergic synaptic

15 actions are robust in the medial nucleus accumbens and the anterolateral dorsal striatum,

16 mediating fast and slow excitation, respectively. Dopamine neuron synaptic actions in

17 cholinergic interneurons are the strongest and most complex, involving all three transmitters,

18 their multiple receptors, and are the most regionally heterogeneous. The caudal striatum forms a

19 single domain with overall weak dopamine neuron synaptic actions. This synaptic mapping

20 reveals that dopamine neuron synaptic actions extend across the entire striatum, are regionally

21 heterogeneous and organized in physiological domains, determined mainly by their excitatory

22 actions. 


\section{Introduction}

24 Dopamine (DA) neurons connect principally with striatal (Str) neurons to control movement,

25 cognition, and motivated behavior. Alterations in DA release underpin major neuropsychiatric

26 disorders and are targeted for their pharmacotherapy. DA neurons in the ventral midbrain project

27 to the Str topographically. More medial ventral tegmental area (VTA) neurons project to the

28 ventral Str or nucleus accumbens (NAc), while laterally located substantia nigra (SN) neurons

29 project to the dorsal Str or caudate-putamen (CPu) (Haber et al., 2000; Ikemoto, 2007).

30 Genetically-specified intersectional strategies have revealed that distinct subclasses of DA

31 neurons project differentially to Str subregions (Heymann et al., 2020; Kramer et al., 2018;

32 Poulin et al., 2018; Wu et al., 2019). Despite regionally heterogeneous functions, the Str has a

33 remarkably uniform cytoarchitecture, comprising about 95\% spiny projection neurons (SPNs)

34 and 5\% interneurons. SPNs are GABAergic and equally divided in two types: SPNs expressing

35 DA D1 receptors (D1R) that project directly to the ventral midbrain (direct-pathway SPNs;

$36 \mathrm{dSPNs}$, and SPNs expressing DA D2R that project to the ventral midbrain via the pallidum

37 (indirect-pathway SPNs; iSPNs) (Gerfen and Surmeier, 2011). Str interneurons comprise

38 cholinergic interneurons (ChIs) and GABAergic interneurons. While ChIs comprise a single

39 type, GABAergic interneurons comprise multiple types; the most distinctive type are fast-spiking

40 interneurons (FSIs) expressing parvalbumin (PV) (Tepper et al., 2018).

41 DA neurons signal via volume transmission (Sulzer et al., 2016), from varicosities with active

42 zone-like release sites lacking postsynaptic elements (Liu and Kaeser, 2019). However, brain

43 slice studies have also revealed that DA neurons have fast synaptic actions in some striatal

44 regions mediated by three transmitters, DA, glutamate and GABA, and can directly excite or

45 inhibit postsynaptic neurons. Glutamate cotransmission requires vesicular glutamate transporter 
46 VGLUT2 (Dal Bo et al., 2004; Hnasko et al., 2010), which is mainly expressed in DA neurons in

47 the medial VTA (Morales and Margolis, 2017). These VGLUT2-expressing DA neurons project

48 strongly to the medial shell of the NAc (Mingote et al., 2019; Poulin et al., 2018), where they

49 invariably elicit excitatory synaptic responses via glutamate cotransmission (Stuber et al., 2010;

50 Tecuapetla et al., 2010). DA neurons in the SN projecting to the $\mathrm{CPu}$ elicit synaptic DA

51 (Chuhma et al., 2014), GABA (Tritsch et al., 2012) and glutamate (Chuhma et al., 2018)

52 responses. GABA cotransmission requires vesicular monoamine transporter VMAT2 (Tritsch et

53 al., 2012) and plasma membrane GABA transporter GAT1 (Tritsch et al., 2014). These sub-

54 second synaptic responses mediated by the three neurotransmitters engage five receptors:

55 ionotropic glutamate receptors (iGluR), metabotropic glutamate receptor 1 (mGluR1), DA D2R,

56 DA D1/5 (D1-like) R, and GABA $\mathrm{R}$ (Cai and Ford, 2018; Chuhma et al., 2014; Chuhma et al.,

57 2018; Kim et al., 2015; Straub et al., 2014; Stuber et al., 2010; Sulzer et al., 1998; Tecuapetla et

58 al., 2010; Tritsch et al., 2012; Wieland et al., 2014).

59 DA neuron synaptic transmission has only been examined in limited locations in the Str, so it has

60 yet to be resolved whether DA neurons have widespread synaptic actions and how they are

61 organized. Here we mapped DA neuron synaptic actions in identified Str neurons by recording

62 direct synaptic responses from identified Str neurons. This has revealed that DA neuron synaptic

63 actions extend across the entire Str and are organized in physiological domains. 


\section{Results}

\section{DA neuron synaptic currents evoked in principal striatal neuron types}

66 To map DA neuron synaptic responses, we stimulated DA neuron axons impinging on the four

67 principal Str neuron types, dSPNs, iSPNs, ChIs, and FSIs (Fig. 1). We used brain slices from

68 triple mutant mice expressing channelrhodopsin 2 (ChR2) in DA neurons driven by the DA

69 transporter (DAT ${ }^{\mathrm{IREScre}}$; floxSTOP-ChR2 mice), and Str cell-type specific markers, D1-tdTomato

70 for dSPNs, D2-enhanced green fluorescent protein (EGFP) for iSPNs, choline acetyltransferase

71 (ChAT)-EGFP for ChIs, and PV-tdTomato for FSIs. While DA neurons in the dorsal raphe may

72 also express ChR2, they do not project to the Str (Lin et al., 2020), so the evoked synaptic

73 responses in the Str arise from ventral midbrain DA neurons. Since D2Rs are expressed in

74 iSPNs, and also in ChIs (Lim et al., 2014), ChIs might express EGFP. However, in D2-EGFP

75 mice only $16.5 \%$ of ChAT positive $\left(\mathrm{ChAT}^{+}\right)$neurons showed EGFP fluorescence

76

77

78

79

80

81

82

83

84

85

86

(Supplemental Fig. 1A, B), which was significantly dimmer than in ChAT negative $\left(\mathrm{ChAT}^{-}\right)$

neurons (Welch's t-test, $\mathrm{t}=-10.3, \mathrm{p}<0.0001$ ) (Supplemental Fig. 1C). ChAT $^{+}$neurons had a larger soma diameter than $\mathrm{ChAT}^{-}$neurons regardless of EGFP fluorescence (one-way ANOVA, $\left.\mathrm{F}_{(2,46)}=88.8, \mathrm{p}<0.0001\right)($ Supplemental Fig. 1D). Therefore, in D2-EGFP mice smaller neurons with brighter EGFP fluorescence were identified as iSPNs.

We photostimulated DA neuron axon terminals with single pulses of blue light, illuminating the entire field of view ( $5 \mathrm{msec}$ duration, at $0.1 \mathrm{~Hz}$ ), at maximum intensity, and recorded synaptic currents in Str neurons, clamping the membrane at -70 mV. Single-receptor type PSCs were pharmacologically isolated by bath application of a cocktail of antagonists to block other receptors: CNQX $40 \mu \mathrm{M}$ and D-APV $100 \mu \mathrm{M}$ for iGluRs, JNJ16259685 $5 \mu \mathrm{M}$ for mGluR1, SCH23390 $10 \mu \mathrm{M}$ for D1/5R, sulpiride $10 \mu \mathrm{M}$ for D2R, and SR95531 (gabazine) $10 \mu \mathrm{M}$ for 


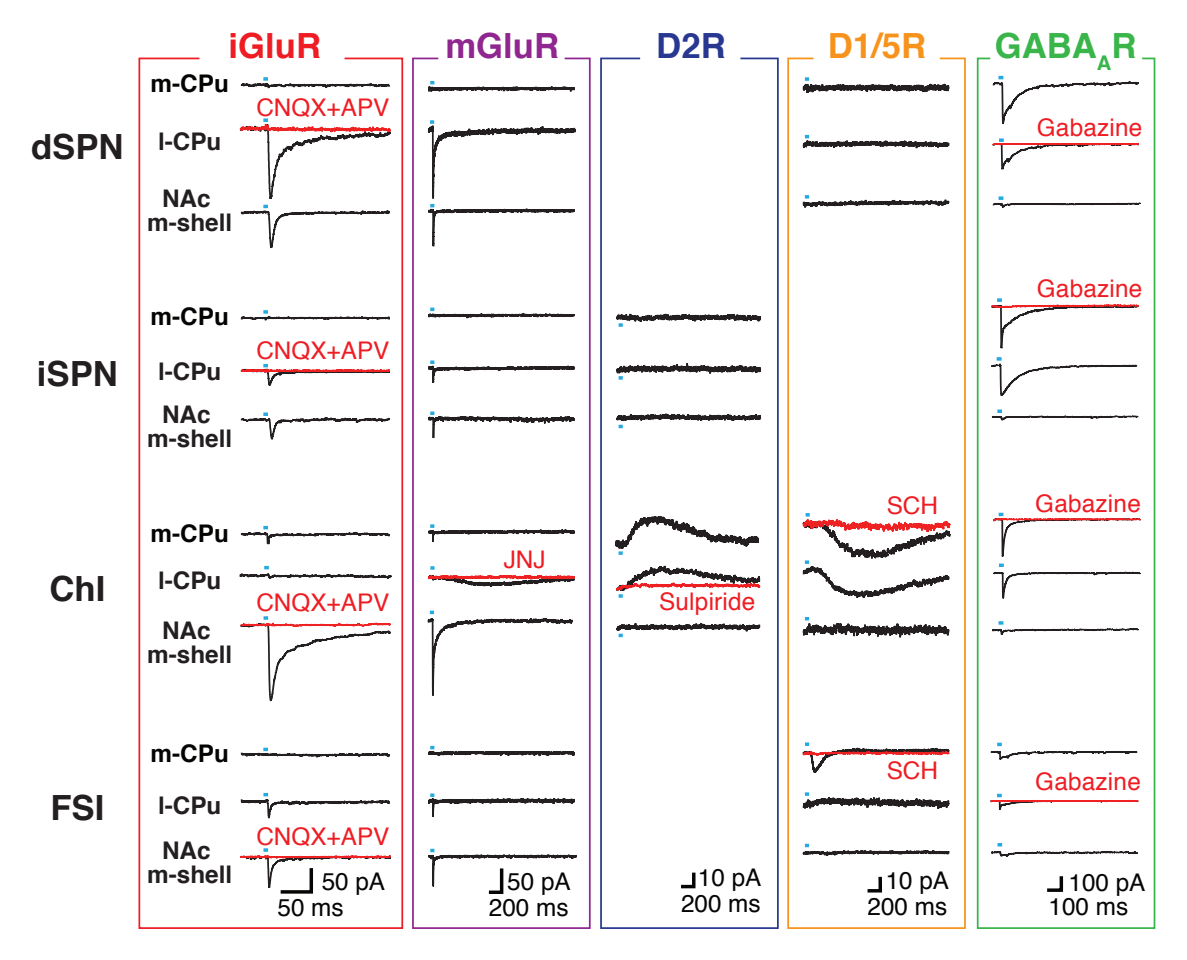

Fig 1

Dopamine-neuron evoked postsynaptic currents in striatal neurons.

In DAT-iresCre;Ai32 mice with dopamine neurons made photoactivatable by expression of channelrhodopsin, pulse illumination (blue bar) of their terminals in striatal brain slices evoked synaptic currents in the four major types of striatal neurons, mediated by five different neurotransmitter receptors, that showed significant regional variation. Recorded striatal cell types were direct-pathway spiny projection neurons (dSPN), indirect-pathway SPN (iSPN), cholinergic interneurons (ChI), and fast-spiking GABAergic interneurons (FSI). Pharmacologically isolated postsynaptic currents (PSCs) were mediated by glutamate via iGluR and mGluR, dopamine via D2R and $\mathrm{D} 1 / 5 \mathrm{R}$, and GABA via $\mathrm{GABA}_{\mathrm{A}} \mathrm{R}$. The three recorded regions were the nucleus accumbens medial shell (NAc m-shell), the medial caudate-putamen $(\mathrm{m}-\mathrm{CPu})$ and lateral caudate-putamen $(1-\mathrm{CPu})$. The same traces are shown for iGluR and mGluR, but on different time scales. PSCs were blocked by the respective antagonist for each transmitter receptor (red traces). D2R responses in dSPNs and FSIs, and D1/5R in iSPNs were not recorded, as the cells lack the corresponding receptors. 
$87 \mathrm{GABA}_{\mathrm{A}} \mathrm{R}$. In all recordings, nicotinic and muscarinic acetylcholine receptors (nAChRs, mAChRs) were blocked with mechamilamine $(10 \mu \mathrm{M})$ and scopolamine $(2 \mu \mathrm{M})$ to eliminate contributions from varying levels of $\mathrm{ChI}$ activity. $\mathrm{A} \mathrm{Cs}^{+}$-based pipette solution was used for glutamate and $\mathrm{GABA}_{\mathrm{A}}$ responses to improve space clamp, while a $\mathrm{K}^{+}$-based pipette solution was

91 used for DA responses, so as not to block $\mathrm{K}^{+}$channels. $\mathrm{GABA}_{\mathrm{A}}$ responses were recorded with

92 high $\mathrm{Cl}^{-}$intracellular solution to obtain inward currents at the same holding potential as for the 93 other responses.

The three DA neuron neurotransmitters elicited PSCs via five types of receptors: iGluRs,

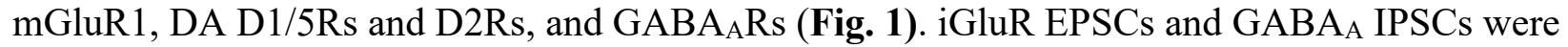
observed in all four cell types, while mGluR EPSCs and D2 IPSCs were observed only in ChIs. D1/5 EPSCs were observed in ChIs and FSIs, but their time courses were different; FSI D1/5 EPSCs showed a faster time course (Rise time 10-90\% peak: ChI 350.0 $\pm 14.7 \mathrm{~ms}$, FSI $34.5 \pm$ $4.9 \mathrm{~ms}, \mathrm{t}=24.2, \mathrm{p}<0.0001 ; \mathrm{ChI} \mathrm{n}=63$ cells, FSI $\mathrm{n}=26$ cells). Thus, only ChIs showed all five

101 types of PSCs. Mediation of each PSC was confirmed by application of the corresponding

102 antagonist (Fig. 2; Fig. 1, red traces). All corresponding antagonist applications blocked the

103 isolated PSCs (Fig. 2; statistics are reported in Supplemental Table 1). ChAT-GFP mice used

104 for identification of ChIs overexpress vesicular ACh transporters (VAChT) (Crittenden et al., 105 2014; Nagy and Aubert, 2012), which might affect PSC measurements, with regional variation.

106 Although AChRs were continuously blocked in all experiments, longer-term modulatory effects 107 of VAChT overexpression might have a regionally different impact on size of PSCs. However, 108 the size distribution of iGluR and mGluR EPSCs in NAc medial shell, medial CPu and lateral $109 \mathrm{CPu}$ was not significantly different in recordings made from ChAT-EGFP mice and non-ChAT- 


\section{$\begin{array}{llll}\text { iGluR } & \text { DGluR } & \text { D1R } & \text { GABA }\end{array}$}
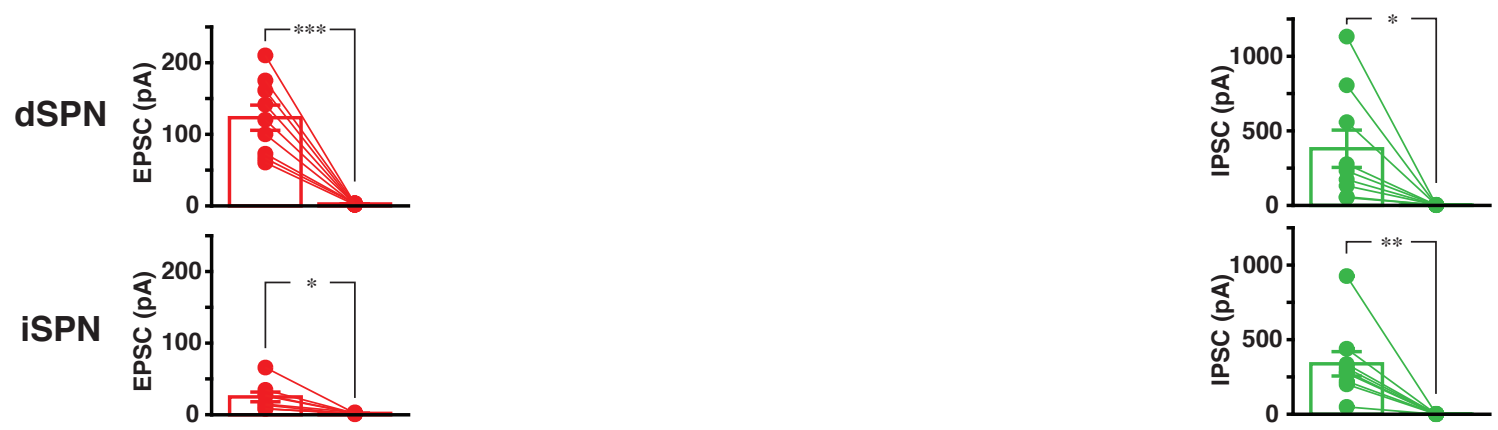

Chl
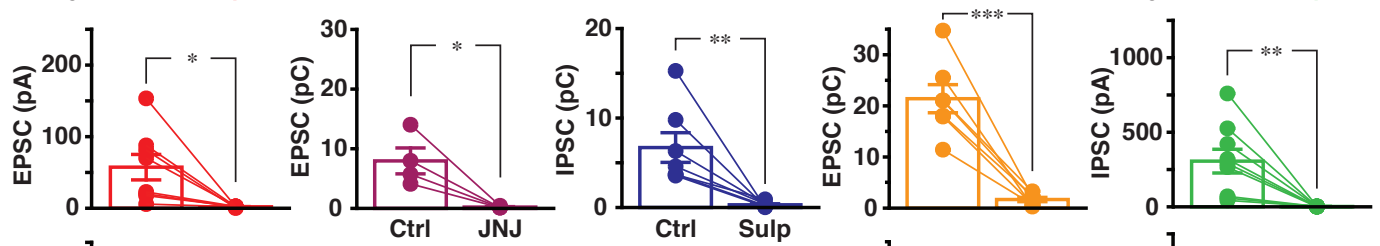

FSI
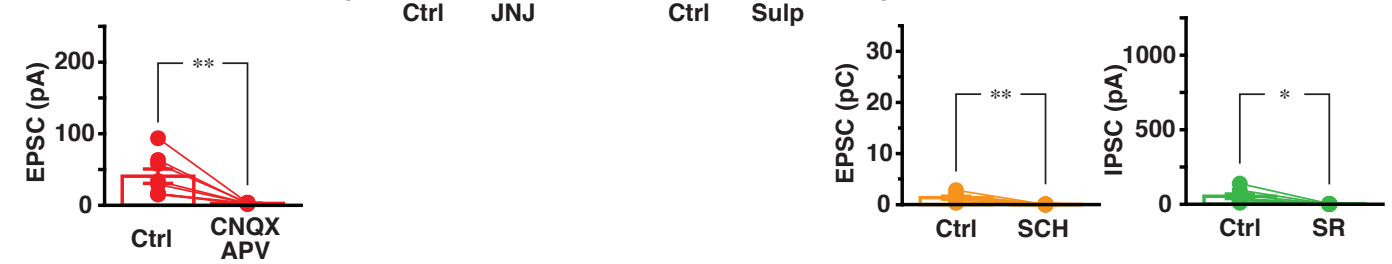

Fig 2

Receptor mediation of pharmacologically isolated PSCs.

Pharmacologically isolated PSCs were blocked by the corresponding receptor antagonist. The antagonists and concentrations used were: iGluR (red) CNQX $40 \mu \mathrm{M}$ and D-APV $100 \mu \mathrm{M}$, mGluR (purple) JNJ16259685 $10 \mu \mathrm{M}, \mathrm{D} 2 \mathrm{R}$ (blue) sulpiride $10 \mu \mathrm{M}, \mathrm{D} 1 / 5 \mathrm{R}$ (orange)

SCH23390 $10 \mu \mathrm{M}$, GABA $_{\mathrm{A}} \mathrm{R}$ (green) SR95531 $10 \mu \mathrm{M}$. PSC size measurements (dots) for control (Ctrl) and antagonist conditions for each cell are connected (lines). Bars show mean \pm S.E.M. Results of paired t-tests are shown in Supplementary Table 1. *,**, and *** indicate p $<0.05, \mathrm{p}<0.01$ and $\mathrm{p}<0.001$, respectively. See Table S1 for parameters of statistics. 
110 EGFP mice, in which ChIs were identified by size and physiology, confirmed by post-recording

111 ChAT immunostaining (two-way ANOVA, genotype effects: iGluR $\mathrm{F}_{(1,57)}=0.14, \mathrm{p}=0.71$;

112 mGluR $\mathrm{F}_{(1,57)}=0.05, \mathrm{p}=0.82$; interaction: $\operatorname{iGluR~} \mathrm{F}_{(2,57)}=0.16, \mathrm{p}=0.85 ; \mathrm{mGluR} \mathrm{F}_{(2,57)}=0.04$,

$113 \mathrm{p}=0.96)$ (Supplemental Fig. 2). Dendrite lengths of ChIs and glutamate EPSC sizes were not

114 correlated (linear regression, iGluR $\mathrm{R}^{2}=0.0002, \mathrm{p}=0.96 ; \mathrm{mGluR}^{2}=0.006, \mathrm{p}=0.76$ )

115 (Supplemental Fig. 3), indicating that differences in the truncation of dendrites during slicing

116 did not contribute to regional differences in PSCs.

117 We then recorded DA neuron synaptic responses in Str neurons throughout the Str, from a

118 minimum of 100 cells for each response type. For iGluR and GABA $\mathrm{R}$ PSCs, peak amplitudes

119 were measured. For mGluR, D2R, D1/5R PSCs in ChIs charge transfer was measured in a 1.5

120 sec window. For the faster D1/5R PSCs in FSIs, we measured charge transfer in a $0.3 \mathrm{sec}$

121 window. PSC sizes were color-scaled and plotted at the Str recording locations to reveal the

122 spatial distribution (Fig. 3). iGluR EPSCs and GABAA IPSCs were observed in all four cell

123 types, but their distributions were different. iGluR EPSCs had two hotspots; the medial NAc in

124 all four cell types and antero-lateral $\mathrm{CPu}$ in dSPNs, iSPNs, and FSIs. Averaged sizes of iGluR

125 EPSCs, including cells without recognizable responses, were larger in dSPNs and ChIs,

126 compared to iSPNs and FSIs (dSPN $24.6 \pm 2.6 \mathrm{pA}, \mathrm{n}=153$ cells; iSPN $11.5 \pm 1.2 \mathrm{pA}, \mathrm{n}=149$

127 cells; ChI $20.7 \pm 2.9$ pA, $\mathrm{n}=133$ cells; FSI $12.2 \mathrm{pA}, \mathrm{n}=115$ cells; one-way ANOVA, $\mathrm{F}_{(3,3546)}=$

$1288.3, \mathrm{p}<0.0001)$. GABA IPSCs did not show clear hotspots but were weaker in the more caudal

129 Str and weak throughout the Str in FSIs (dSPN 158.1 \pm 17.0 pA, $\mathrm{n}=138$ cells; iSPN $119.0 \pm 18.0$

$130 \mathrm{pA}, \mathrm{n}=133$ cells; ChI $211.8 \pm 22.5 \mathrm{pA}, \mathrm{n}=120$ cells; FSI $31.8 \pm 4.4 \mathrm{pA}, \mathrm{n}=108$ cells; one-way

131 ANOVA, $\left.\mathrm{F}_{(3,3495)}=16.9, \mathrm{p}<0.0001\right)$. mGluR EPSCs were observed only in the anterolateral

$132 \mathrm{CPu}$ in ChIs. DA responses were not observed in SPNs, despite the high-expression of D1Rs in 


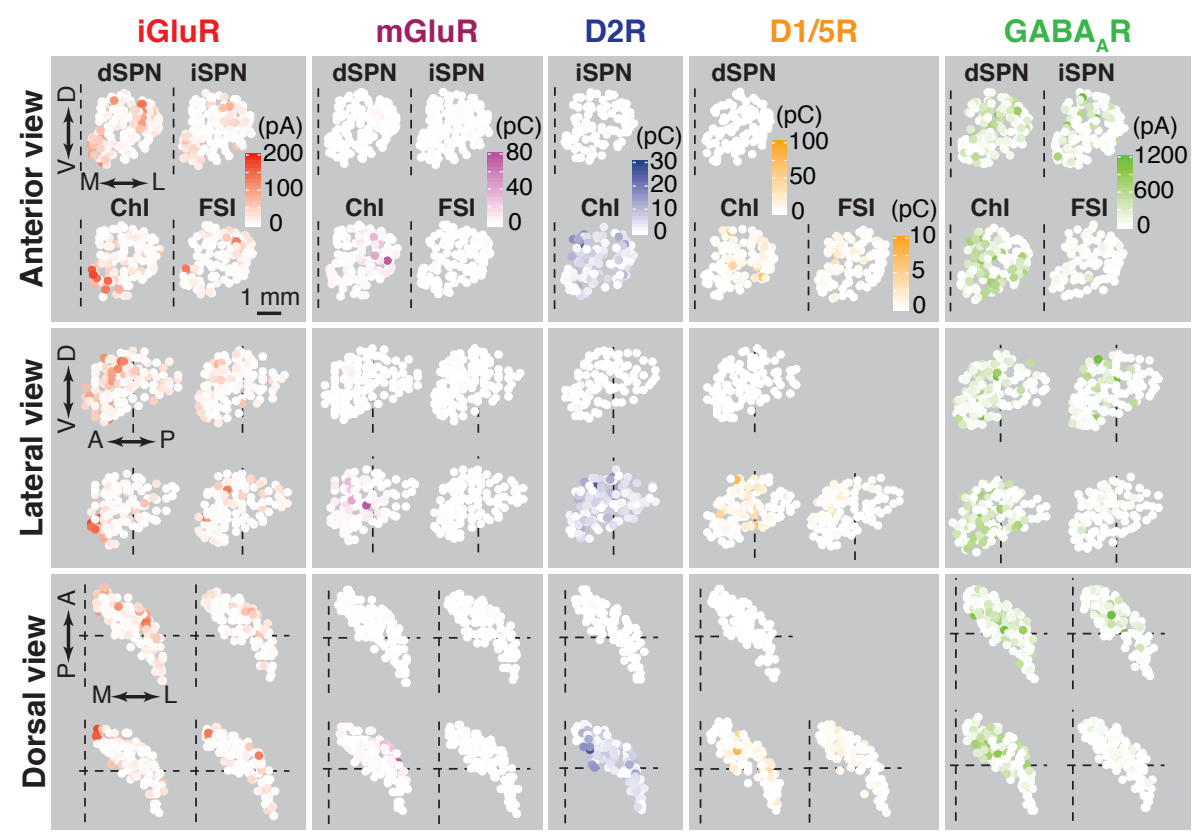

Fig 3

Distribution of dopamine-neuron evoked postsynaptic currents across the striatum.

The size of PSCs, reported as amplitude for inotropic responses (iGluR, $\mathrm{GABA}_{\mathrm{A}} \mathrm{R}$, in $\mathrm{pA}$ ) or charge transfer for metabotropic responses (mGluR, $\mathrm{D} 2 \mathrm{R}, \mathrm{D} 1 / 5 \mathrm{R}$, in $\mathrm{pC}$ ), are plotted on a three-dimensional (3D) grid subtending the left striatum. Each recorded cell, indicated by a small sphere, is color coded by the size of the recorded PSC, with color intensity in red (iGluR), purple (mGluR), blue (D2R), orange (D1/5R), or green (GABAAR). Anterior (top), lateral (middle), and dorsal (bottom) views are shown; arrows indicate ventral (V), dorsal (D), medial (M), lateral (L), anterior $(\mathrm{A})$, and posterior $(\mathrm{P})$ directions. Dashed lines indicate bregma zero. D1/5R responses in FSIs are shown on a different color scale. PSCs were recorded in 1603 striatal cells: Glu dSPN 153, iSPN 149, ChI 133, FSI 115; D2 iSPN 101, ChI 121; D1/5 dSPN 104, ChI 121, FSI 107; GABAA dSPN 138, iSPN 133, ChI 120, FSI 108. 
133 dSPN and D2Rs in iSPNs (Kreitzer, 2009; Surmeier et al., 2011). Indeed, charge transfers in

134 SPNs measured as DA PSCs did not differ from baseline charge transfer recorded without

135 photostimulation (two-sample Kolmogorov-Smirnov test; dSPN p =0.48; iSPN p =0.42), while

136 D2R IPSCs in ChIs (positive control) showed a significant difference $(\mathrm{p}<0.0001)$

137 (Supplemental Fig. 4). D2R IPSCs were observed widely in ChIs, stronger in the medial CPu

138 and weaker in the medial NAc. D1/5R EPSCs were widely observed, both in ChIs and FSIs,

139 without clear hotspots. D1/5R EPSC charge transfers in FSIs were smaller due to their faster

140 time course.

141 Maps of DA neuron synaptic currents in the striatum

142 To generate continuous maps of PSC size, we interpolated individual measurements on a $150 \mu \mathrm{m}$

143 cubic grid (4177 points in the unilateral Str) by kriging, a geostatistical method of estimation

144 based on relatively limited sample numbers, using weighted averages (Matheron, 1963 1246-

145 1266). This method assumes spatial autocorrelation; closer points are similar, while distant

146 points are different (Bivand et al., 2013), and the weights for averaging are obtained based on

147 sampled data. When semivariances of PSC sizes for any two locations were calculated and

148 plotted against distance between them (experimental variogram), two points that were closer had

149 smaller semivariance for all response types (Supplemental Fig. 5), showing spatial

150 autocorrelation. Weights for averaging in kriging were obtained from the experimental

151 variograms fitted by covariance functions (Supplemental Fig. 5; fitting parameters are in

152 Supplemental Table 2).

153 We plotted estimated PSC sizes for each receptor type in the Str. Anterior, lateral, and dorsal

154 views of surfaces of the 3D plots are shown in Fig. 4; coronal sections of the 3D plots are shown

155 in Fig. 5 for iGluR, mGluR, and D2R and in Fig. 6 for D1/5R and GABA $\mathrm{R}$ PSCs. The 


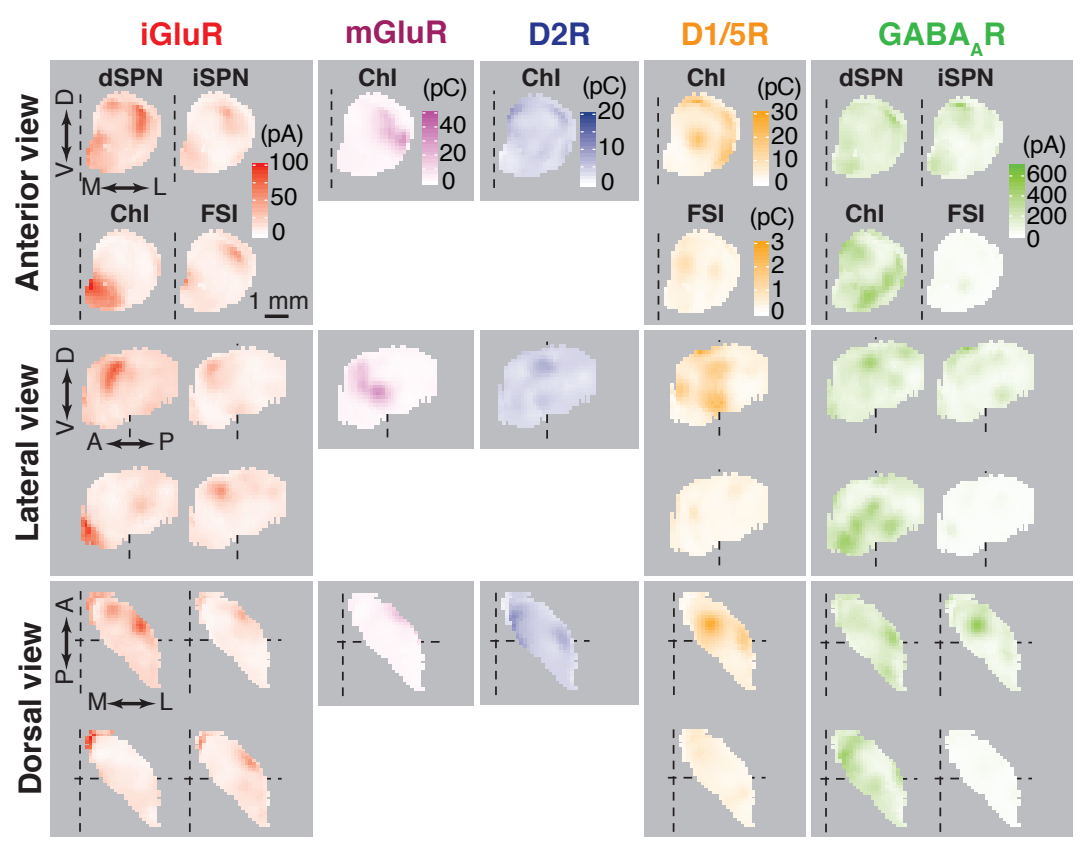

Fig 4

Maps of estimated dopamine-neuron evoked postsynaptic currents in 3D.

Maps of estimated PSC size on a $\sim 150 \mu \mathrm{m} 3 \mathrm{D}$ grid were made based on recorded PSC data using geostatistical methods. Only cell type/receptor recordings with significant responses were mapped. Maps maintain the same color scheme and layout as Fig. 3. 
156 continuous maps showed response hotspots more clearly than the raw data plots. In iGluR PSC

157 maps, all four cell types showed a hotspot in the medial NAc, although the intensity and

158 distribution differed among cell types (Fig.4; Fig. 5). The dSPN, iSPN, and FSI hotspots were

159 limited to the medial shell, while the ChI hotspot extended to almost the entire NAc. Another

160 iGluR hotspot was seen in the anterolateral CPu for dSPNs, iSPNs, and FSIs, but not ChIs.

161 Although FSIs showed both the medial NAc and antero-lateral CPu hotspots, the hotspots were

162 smaller than those for the other cell types. Estimated iGluR responses in the caudal Str (caudal to

163 bregma, bottom of Fig. 5) had recognizable sizes, but clear hotspots were not observed in all four

164 cell types. In contrast to iGluR maps, mGluR responses were seen only in ChIs in one hotspot in

165 the anterolateral CPu (Fig. 4; Fig. 5). DA D2R responses were widely observed throughout the

166 Str, with a weak response spot in the NAc medial shell and a hotspot in the dorsomedial $\mathrm{CPu}$

167 (Fig. 4; Fig. 5). Despite the lack of clear hotspots for D1/5R or GABA $A_{A}$ responses in the raw

168 data plots (Fig. 3), the estimated response maps showed some spatial patterns (Fig. 4; Fig. 6).

$169 \mathrm{D} 1 / 5 \mathrm{R}$ responses in ChIs formed an oblong hotspot in the $\mathrm{CPu}$ near bregma, and a second

170 hotspot in the middle of the anterior $\mathrm{CPu}(\mathrm{Fig}$. 4). Coronal sections revealed that the oblong

171 hotspot and the anterior $\mathrm{CPu}$ hotspots were connected, and also revealed weak response areas in

172 the medial CPu and NAc (Fig. 6). In FSIs, D1/5R responses were more prominent in the medial

$173 \mathrm{CPu}($ Fig. 6). Overall GABA $\mathrm{R}$ responses were weaker in FSIs compared to the other three cell

174 types. In SPNs and ChIs, GABA $\mathrm{A}_{\mathrm{R}}$ responses tended to be larger in the anterior Str, and weaker

175 in the most caudal $\operatorname{Str}(<-1.0 \mathrm{~mm}$ from bregma) (Fig. 6). In SPNs, the middle part of the anterior

176 Str showed larger $\mathrm{GABA}_{\mathrm{A}}$ responses, and the anterolateral $\mathrm{CPu}$ showed weaker responses. In

177 ChIs, the medial NAc and anterior dorsolateral $\mathrm{CPu}$ showed weaker $\mathrm{GABA}_{\mathrm{A}}$ responses, as well

178 as the caudal-most Str. 


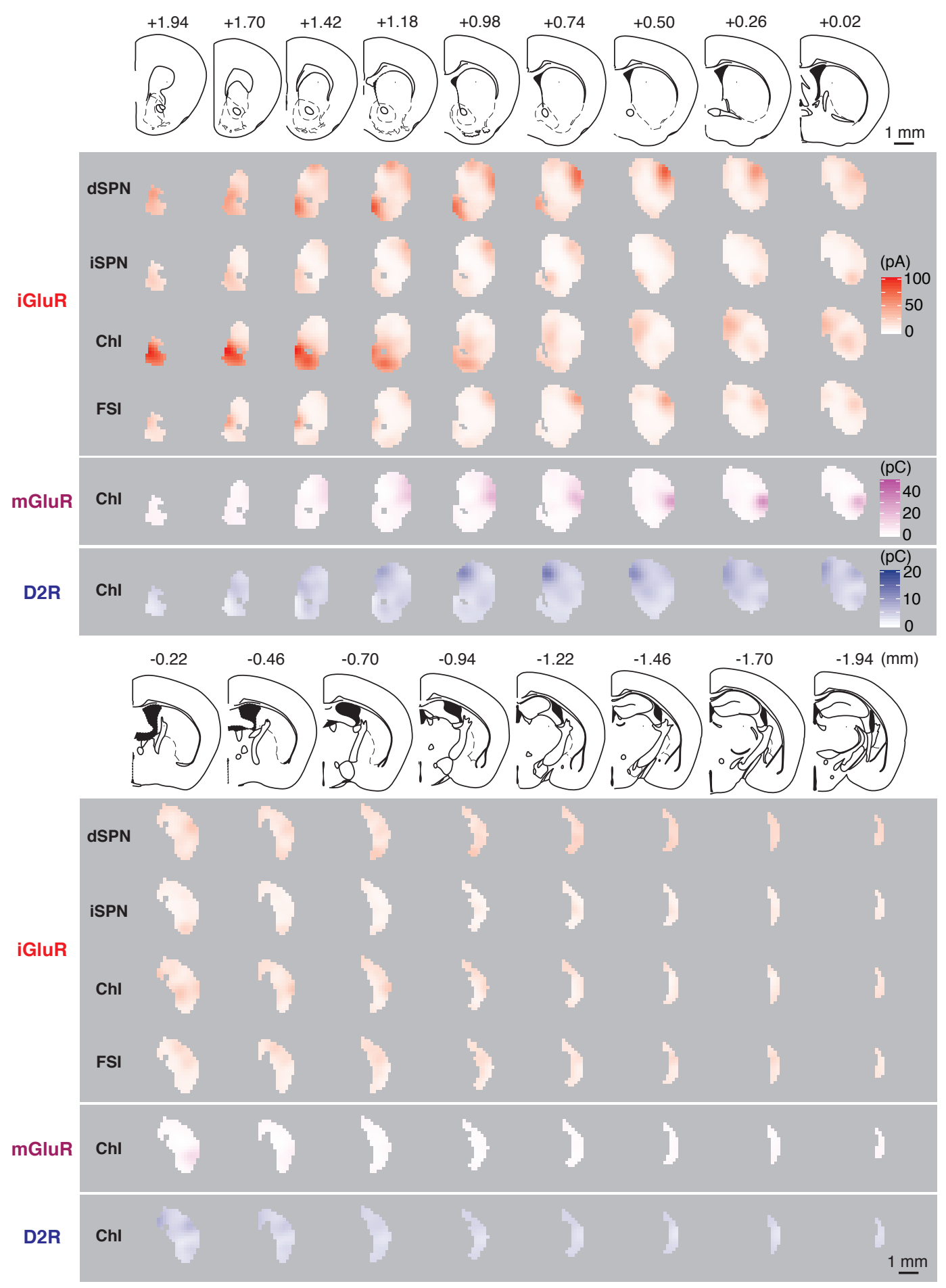

Fig 5

Maps of estimated dopamine-neuron evoked iGluR, mGluR, and D2R PSCs in coronal sections.

Estimated iGluR (red), mGluR (purple), and D2R (blue) PSC sizes in the Str in coronal sections every $\sim 250 \mu \mathrm{m}$ are shown, corresponding to every other left-sided slice in mouse atlas. Anterior-posterior location from bregma and unilateral brain atlas sections are shown above. 


\section{DA neuron synaptic connections define physiological striatal domains}

180 To identify Str subregions based on synaptic connectivity for all observed transmitter responses

181 in each Str cell type, we performed a cluster analysis for each cell type (Fig. 7) and for each

182 transmitter (Fig. 8), using hierarchical clustering with spatial constraint (Chavent et al., 2018),

183 mixing location and data (response size) matrices. PSC sizes were normalized to the maximum

184 and minimum values of each map before clustering. Optimal numbers of clusters were

185 determined by the silhouette method (Rousseeuw, 1987). In each cluster, we calculated mean and

186 standard deviation (SD) of each response type, and the number of grid points as an estimate of

187 cluster size (Supplemental Table 3; Supplemental Table 4). We also calculated cluster-

188 separation $\mathrm{F}$ values, the ratio of between-cluster variance to within-cluster variance (Calinski and

189 Harabasz, 1974), for each type of response to identify principal determinants of the clusters

190 (Supplemental Table 3; Supplemental Table 4). Larger F values indicate better separation of

191 clusters, and that the response type contributes more to the clustering.

192 In cell-type clusters for both types of SPNs, the optimal number of clusters was three: the medial

193 NAc and anterolateral $\mathrm{CPu}$ formed one cluster with the strongest iGluR PSCs, a second cluster in

194 the posterior $\mathrm{CPu}$ where both iGluR and $\mathrm{GABA}_{\mathrm{A}} \mathrm{R}$ PSCs were weak, and a third cluster that

195 comprised the rest of the Str (Fig. 7). However, the posterior $\mathrm{CPu}$ cluster in iSPNs extended

196 more anteriorly than in dSPNs, over most of the lateral CPu. In dSPNs, the iGluR F was

197 substantially larger than the $\mathrm{GABA}_{\mathrm{A}} \mathrm{R} F$, suggesting that clusters were determined more by

198 iGluR responses (Supplemental Table 3). In iSPNs, the GABA $\mathrm{R} F$ was larger, but the

199 difference between iGluR and $\mathrm{GABA}_{\mathrm{A}} \mathrm{R}$ was not as large as in dSPNs, suggesting that both

$200 \mathrm{iGluR}$ and $\mathrm{GABA}_{\mathrm{A}} \mathrm{R}$ responses contributed equally to the clustering (Supplemental Table 3). In

201 ChIs, all five types of responses were observed and the optimal number of clusters was six (Fig. 
7). The medial NAc cluster and anterolateral $\mathrm{CPu}$ cluster were characterized by stronger

excitatory responses; iGluR in the former, and mGluR and D1/5R in the latter. The anteromedial

$204 \mathrm{CPu}$ formed a distinct cluster, where inhibition was dominant due to the strongest D2R and

$205 \mathrm{GABA}_{\mathrm{A}} \mathrm{R}$ responses. The caudal $\mathrm{CPu}$ showed generally small responses, as observed in SPNs.

206 iGluR and GABA $\mathrm{R}$ responses in ChIs were the largest among those in the four cell types

207 (Supplemental Table 3). The most prominent determinant of clusters in ChIs was iGluR

208 followed by D2R and GABA $\mathrm{R}$ responses (Supplemental Table 3). In FSIs, although all

209 responses were small, there were five clusters: a dorsomedial NAc and anterolateral CPu cluster

210 with larger iGluR and $\mathrm{GABA}_{\mathrm{A}} \mathrm{R}$ PSCs, an anterior dorsomedial CPu cluster with the strongest

211 D1/5R responses, and a caudal CPu cluster with weak responses (Fig. 7). The strongest

212 determinant of FSI clusters was D1/5R responses (Supplemental Table 3).

213 Transmitter clusters confirmed glutamate hotspots observed in cell-type clusters (Fig. 8). When

214 clustering was done with all types of glutamate responses in all cell types (glutamate clusters),

215 the optimal number of clusters was four (Fig. 8). Among them, two clusters were highlighted;

216 the medial NAc characterized by iGluR responses in ChIs and dSPNs, and the anterolateral CPu

217 characterized by mGluR responses in ChIs and iGluR responses in dSPNs. The clusters were

218 determined by ChI iGluR, dSPN iGluR, and ChI mGluR PSCs, according to F values

219 (Supplemental Table 4). In clustering of DA responses, the optimal number of clusters was

220 four: medial NAc and posterior $\mathrm{CPu}$ clusters with weaker DA responses, a medial $\mathrm{CPu}$ cluster

221 with prominent D2R responses, and an anterolateral CPu cluster with prominent D1/5R

222 responses (Fig. 8). DA clusters were determined mostly by D1/5R responses, suggesting that

223 D2R responses are distributed more widely and uniformly and contribute less to the clustering

224 (Supplemental Table 4). The optimal number of GABA clusters was four, and GABA clusters 


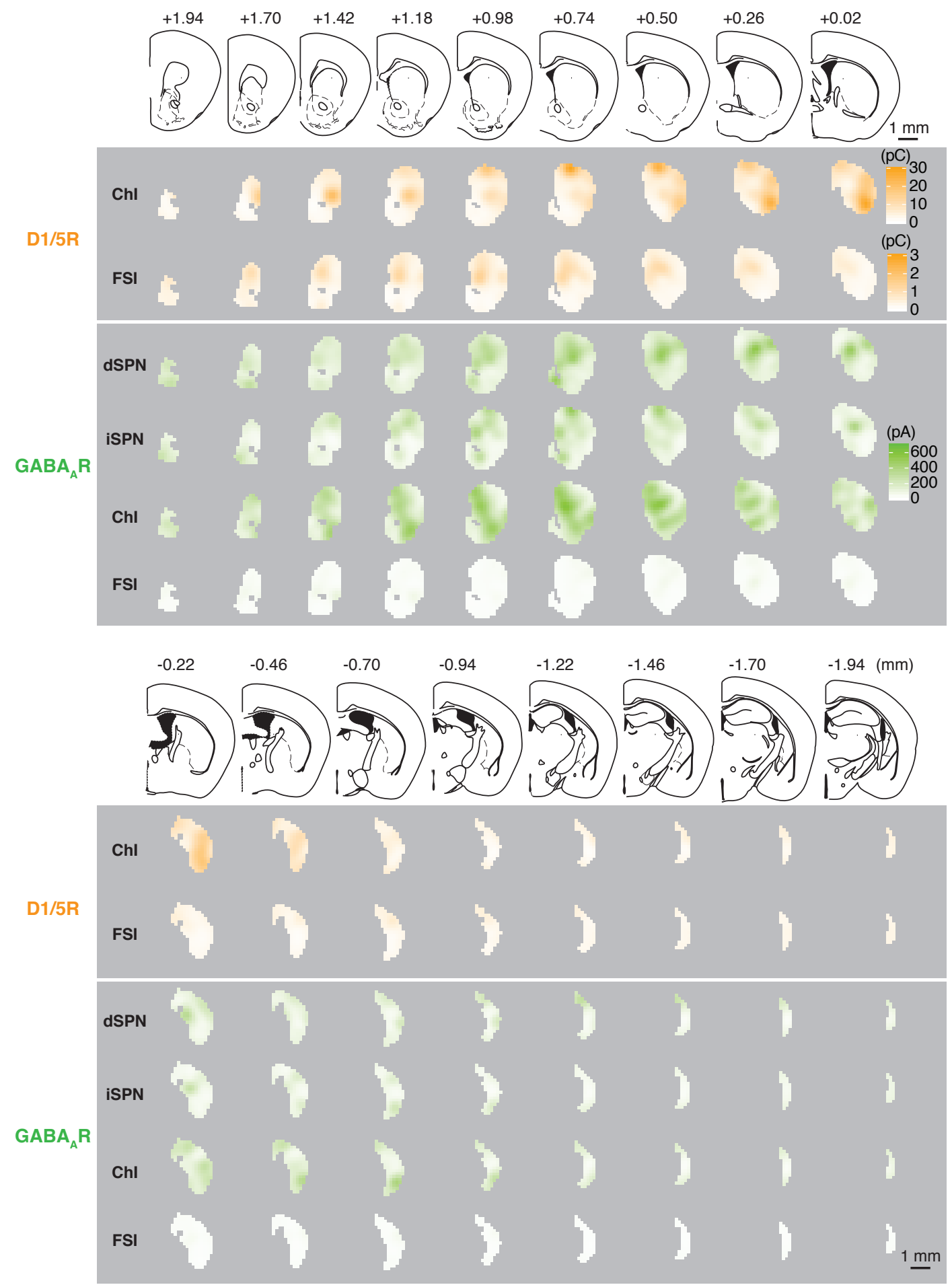

Fig 6

Maps of estimated dopamine-neuron evoked D1/5R and GABAAR PSCs in coronal sections.

Estimated D1/5R (orange) and $\mathrm{GABA}_{\mathrm{A}} \mathrm{R}$ (green) PSC sizes in the Str in coronal sections are shown with the same scheme as Fig 5. 


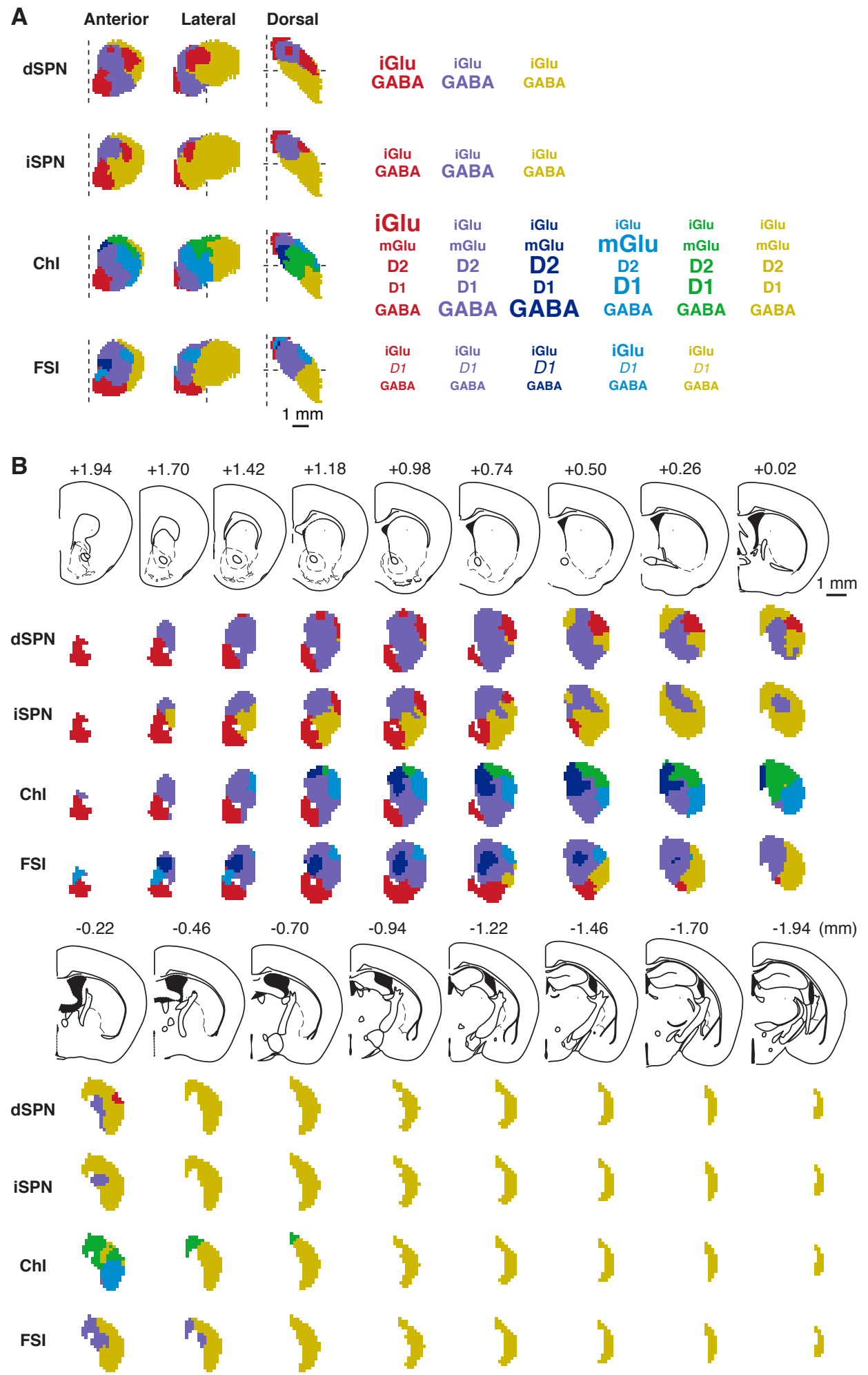

Fig 7

Domains of dopamine-neuron evoked PSCs by Str cell-type.

Clusters by striatal cell type based on estimated PSC sizes are shown in anterior, lateral, and dorsal 3D views (A) and in coronal sections (B). Right panel in A shows mean PSC size for each cluster scaled by font size relative to the maximum response for each PSC type. D1/5R PSCs in FSIs are italicized to indicate that they were measured on a different scale than in ChIs. 
A

A

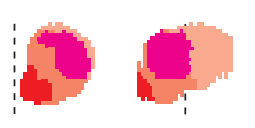

DA
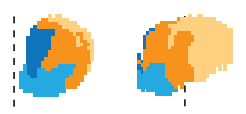

GABA
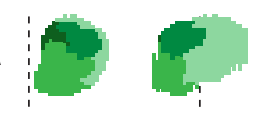

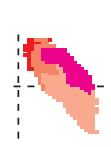

dSPN iGlu iSPN iGlu

Chl iGlu

FSI iGlu

Chl mGlu

dSPN iGlu

iSPN iGlu

Chl iGlu

FSI iGlu

Chl mGlu

dSPN iGlu

iSPN iGlu

dSPN iGlu

Chl iGlu

FSI iGlu

ChliGlu

FSI iGlu

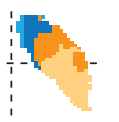

\section{ChI D2}

ChI D1

ChI D2

ChI D1

Chl D2

Chl D1

FSID1

Chl D2

FSI D1

FSI 1

dSPN

$\begin{array}{ll}\text { dSPN } & \text { dSPN } \\ \text { iSPN } & \text { iSPN }\end{array}$

Chl Chl

FSI

iSPN

ChI

dSPN

iSPN

Chl

$1 \underline{\mathrm{mm}}$

B
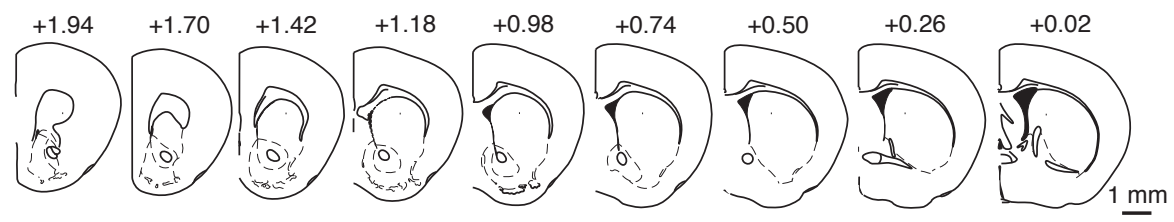

Glu
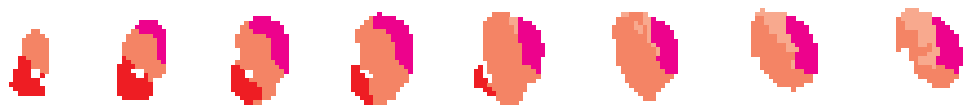

DA
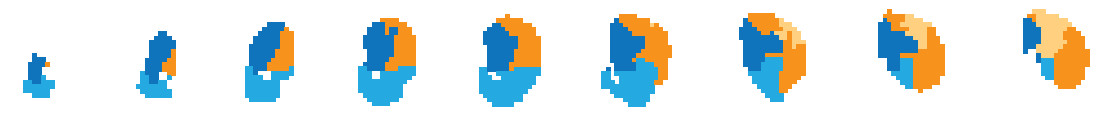

GABA
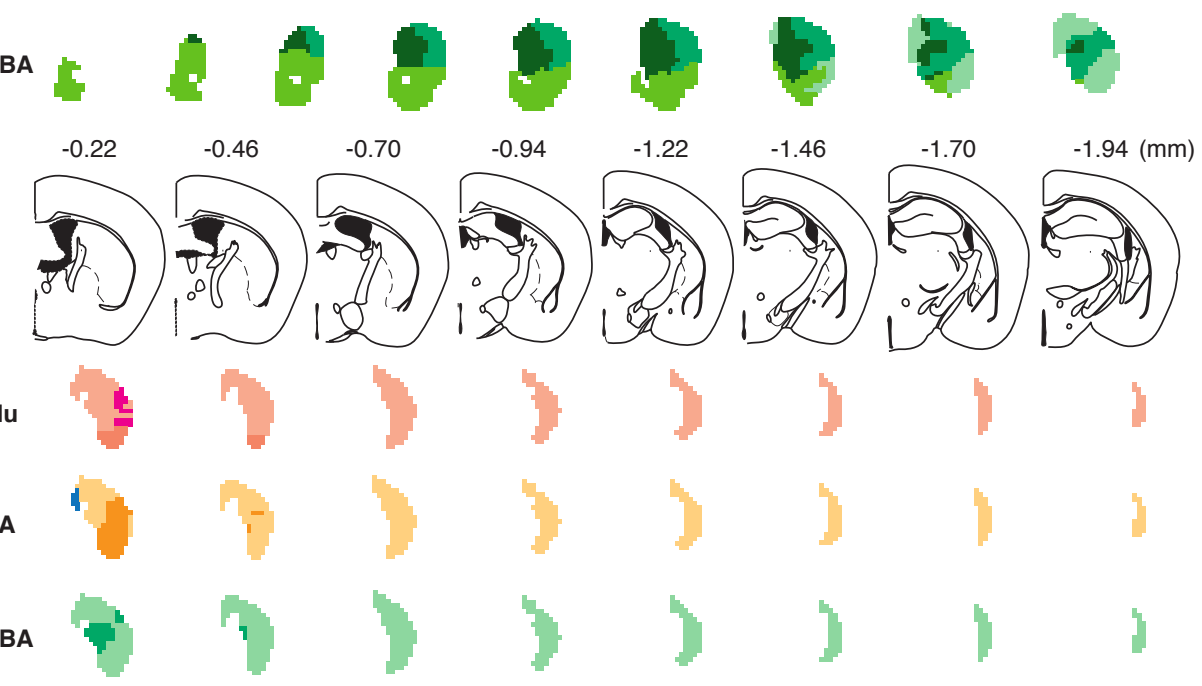

Fig 8

Domains of dopamine-neuron evoked PSCs by neurotransmitter.

Clusters by DA neuron neurotransmitter are shown in anterior, lateral, and dorsal views (A) and in coronal sections (B). Right panel shows mean PSC size for each cluster scaled by font size relative to the maximum response for each PSC type, with the same scheme as Fig 7. 
225 showed the same tendency in all cell types: strongest in the antero-dorsomedial $\mathrm{CPu}$, and

226 weakest in the posterior $\mathrm{CPu}($ Fig. 8). Clusters were determined almost equally by dSPN, iSPN,

227 and ChI, but not by FSIs (Supplemental Table 4). Transmitter clusters supported another

228 observation in the cell-type clustering; in addition to the two glutamate hotspots, the posterior

$229 \mathrm{CPu}$ formed a single cluster with universally weak DA neuron synaptic responses. Taken

230 together, clusters were predominantly determined by glutamate and D1/5R responses, compared

231 to $\mathrm{D} 2 \mathrm{R}$ or $\mathrm{GABA}_{\mathrm{A}} \mathrm{R}$ responses. Both glutamate and DA D1/5 receptors mediate excitatory

232 responses, indicatinging that DA neuron physiological domains in the Str are determined mainly

233 by their excitatory synaptic actions. 


\section{Discussion}

236 Over time, DA neurons have gone from a homogeneous group of neurons recognized by their

237 monoamine content and tyrosine hydroxylase expression, to subpopulations receiving differential

238 inputs, projecting differentially, with diversity in their activity patterns, gene expression,

239 cotransmission and behavioral functions. This heterogeneity appears to extend to DA neuron

240 synaptic actions in the Str. However, the direct synaptic actions of DA neurons have only been

241 identified in some DA neuron projections, in limited Str regions. Here we have mapped DA

242 neuron synaptic actions in the Str, revealing that DA neurons have fast synaptic actions

243 extending across the Str. SPNs show only iGluR and GABA $\mathrm{R}$ responses, despite high

244 expression of DA receptors. FSIs show D1/5R responses in addition to $i G l u R$ and GABA $R$

245 responses. Among the four principal types of Str neurons, only ChIs show responses to the three

246 DA neuron neurotransmitters, mediated by five types of postsynaptic receptors. Maps of PSC

247 size identify the most robust connections in the medial NAc for iGluR responses, anterolateral

$248 \mathrm{CPu}$ for iGluR and mGluR responses, and medial $\mathrm{CPu}$ for $\mathrm{D} 2 \mathrm{R}$ responses. Cell type clustering

249 and transmitter clustering reveal discrete physiological domains: the medial NAc and

250 anterolateral $\mathrm{CPu}$ as excitatory-dominant clusters, and the caudal $\mathrm{CPu}$ as a single cluster with

251 universally weak synaptic connections. Inhibitory responses are distributed more broadly in the

252 Str, so clusters are determined mainly by excitatory glutamate and D1/5R actions.

\section{DA neuron synaptic transmission in the Str}

254 Ventral midbrain DA neurons have two temporal modes of transmission; slower volume

255 transmission (Sulzer et al., 2016), and faster, point-to-point synaptic transmission. DA neuron

256 DA synaptic transmission was first revealed in recordings of dendrodendritic connections

257 between DA neurons in the ventral midbrain (Beckstead et al., 2004). In the Str, DA neuron 
258 glutamate connections were revealed with electrical stimulation of DA neuron axons (Chuhma et

259 al., 2004) and optogenetic stimulation of DA neuron terminals impinging on SPNs in the NAc

260 (Stuber et al., 2010; Tecuapetla et al., 2010). DA neurons made stronger iGluR-mediated

261 connections to ChIs in the NAc m-shell, and direct DA D2R-mediated connections in the dStr

262 (Chuhma et al., 2014). The present results show that fast DA neuron synaptic signaling spans the

263 entire Str. DA neuron synaptic actions are not only faster, but also show cell-type specificity and

264 convey discrete information, with regional heterogeneity due to blending of different types of

265 responses.

266 In addition to fast PSCs mediated by ion channel-type receptors, i.e. iGluR and GABAAR, G-

267 protein coupled receptors also mediate faster signals in Str interneurons. Stimulation of DA

268 neuron axons elicits D2R IPSCs, D1/5 EPSCs , and mGluR EPSCs in ChIs (Cai and Ford, 2018;

269 Chuhma et al., 2014; Chuhma et al., 2018; Straub et al., 2014; Wieland et al., 2014). While Str

270 neurons express $\mathrm{GABA}_{\mathrm{B}}$ receptors, GABAB-mediated PSCs were not observed in any Str cell

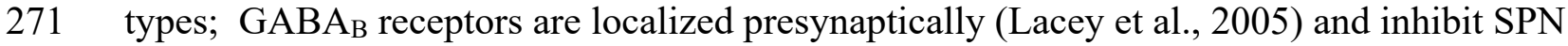

272 collaterals (Shi and Rayport, 1994), and. D1/5R mediated depolarization and inward currents

273 were observed in FSIs with bath application of D1 agonist (Centonze et al., 2003). However,

274 perfusion is not the same as synaptic transmission, so it was not known whether action potential-

275 dependent DA release would elicit D1/5R EPSCs in FSIs. We found that DA neurons elicit D1/5

276 EPSCs in FSIs, while SPNs show only iGluR and GABA $\mathrm{A}_{\mathrm{R}}$ PSCs despite their high DA receptor

277 expression (Kreitzer, 2009; Surmeier et al., 2011). iSPNs show D2R IPSCs following

278 transfection with G-protein coupled inward rectifier $\mathrm{K}^{+}$(GIRK2) channels (Marcott et al., 2014),

279 so the observation of fast DA PSCs in interneurons appears to depend on the expression of

280 effector channels mediating currents, rather than DA receptors alone. Indeed, the D2R response 
281 in GIRK2 transfected iSPNs is slower in its latency and time course than that in ChIs, suggesting

282 that other factors determine the time course of DA synaptic transmission such as the distance

283 from DA release sites to DA receptors.

284 We identified DA neuron synaptic domains by cell-type cluster and transmitter-type cluster

285 analysis. Both types of clusters were mainly determined by excitatory synaptic actions, i.e.

286 glutamate and $\mathrm{D} 1 / 5 \mathrm{R}$ responses, and mostly located in the anterior $\mathrm{CPu}$ (anterior to bregma) and

287 NAc. The posterior CPu (posterior to bregma) formed its own cluster for all four cell types, with

288 comparatively weak connections that showed little variation, indicating that most DA neuron

289 signaling in the posterior $\mathrm{CPu}$ is via volume transmission. Although hotspots of glutamate

290 cotransmission identified in this study correspond to locations of dense projections of glutamate

291 cotransmitting DA neurons identified by intersectional expression of marker proteins (Chuhma et

292 al., 2018; Mingote et al., 2019; Poulin et al., 2018), dense projections are also observed in the

293 most caudal Str (Poulin et al., 2018), which belongs to the posterior CPu cluster, with weak

294 synaptic connections. This discrepancy could be due to fewer release sites per projection fiber in

295 the most caudal Str, as denser projections do not necessarily release more transmitters. Another

296 possibility is that the projections in the caudal CPu synapse on other cell types, i.e. other types of

297 GABA interneurons or glia. This is a potential limitation of the present mapping study, but it

298 does emphasize further that anatomical evidence of connectivity does not translate directly to

299 physiological measures of connectivity.

\section{$300 \quad$ Functional implications}

301 DA neurons target ChIs preferentially and elicit the strongest responses, in contrast to the

302 universally weak responses in FSIs. Considering their shallower resting membrane potential and

303 spontaneous firing (Kawaguchi, 1993; Kreitzer, 2009), ChIs in the medial NAc and anterolateral 
$304 \mathrm{CPu}$ are the most easily excited by DA neuron synaptic inputs. Indeed, excitatory inputs from

305 DA neurons can drive ChIs to fire, despite substantial GABAA input (Chuhma et al., 2014;

306 Chuhma et al., 2018; Straub et al., 2014). Although the two subregions are excited by DA neuron

307 firing, the timing of the excitation differs due to differences in receptor mediation, iGluR versus

$308 \mathrm{mGluR}$ and D1/5R. In other regions, DA neuron synaptic inputs appear to have inhibitory

309 effects, pausing ChIs in the medial $\mathrm{CPu}$ via $\mathrm{D} 2 \mathrm{R}$ and $\mathrm{GABA}_{\mathrm{A}}$ receptors. Thus, DA neurons

310 appear to use ChIs as hubs to control the Str circuitry with profoundly different synaptic effects

311 in different Str subregions.

312 These physiological domains provide a guide to in vivo stimulation location, to study regional

313 functions of the $\mathrm{Str}$, particularly in the $\mathrm{CPu}$. As neighboring DA neurons engage in concerted

314 burst activity (Beeler and Kisbye Dreyer, 2019; Parker et al., 2016), which extends to their

315 terminals (Cai et al., 2020), activation of a subpopulation of terminals mimics physiologically

316 relevant burst activity. So, the physiological domains mapped in this study may correspond to

317 functional units controlled by concerted burst activity of DA neurons. Nonetheless, differences in

318 the coherence of DA neuron activity is likely to differentially affect signal transduction. The Str

319 domains identify representative recording locations necessary for the characterization of DA

320 neuron transmission in the Str, in order to evaluate developmental, maturational and pathological

321 changes. The domains may point to ways to achieve circuit specific pharmacotherapy by

322 combinatorial drug targeting. 


\section{Materials and Methods}

326 Mice

327 Mice were handled in accordance with the guidelines of the National Institutes of Health Guide

328 for the Care and Use of Laboratory Animals, under protocol NYSPI-1494 approved by the

329 Institutional Animal Care and Use Committee of New York State Psychiatric Institute. Mice

330 were group housed and maintained on a 12 hour light/dark cycle. All slice/tissue preparations

331 were done during the light phase. Food and water were supplied ad libitum. A total of 264 mice

332 (124 male and 140 female), 2 to 3 months of age (postnatal days 60 to 91) were used.

333 Mice were C57BL6J/129Sv mixed background, backcrossed more than 5 times to C57BL6J and

334 kept inbred. D2-EGFP mice, originally on a FVB background, were crossed to C57BL6J more

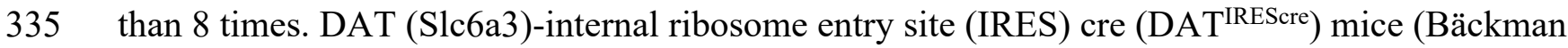

336 et al., 2006) (Jackson Laboratories, Bar Harbor, ME; RRID:IMSR_JAX:006660) were mated

337 with ROSA26-floxSTOP-CAG-ChR2-EYFP (Ai32; ChR2-EYFP) (RRID:IMSR_JAX:024109)

338 to generate mice with selective expression of ChR2 in DA neurons and enable selective

339 stimulation of DA neuron terminals in the Str. Mice were homozygous for ChR2-EYFP to enable

340 reliable stimulation. For identification of dSPN, iSPN, ChI, and FSI Str neurons, mice with

341 fluorescent genetic markers for each neuron type, D1-tdTomato (RRID:IMSR_JAX:016204),

342 D2-EGFP (GENSAT; RRID:MMRRC_000230-UNC), ChAT-EGFP

343 (RRID:IMSR_JAX:007902), or PV-tdTomato (RRID:IMSR_JAX:027395), respectively, were

344 bred with DAT ${ }^{\text {IREScre} ; C h R 2-E Y F P ~ d o u b l e ~ m u t a n t ~ m i c e . ~ F o r ~ r e c o r d i n g ~ f r o m ~ C h I s ~ w i t h ~} \mathrm{~K}^{+}$-based

345 pipette solution, double mutant of DAT ${ }^{\mathrm{IRES}}$;re; ChR2-EYFP mice without ChAT-EGFP were

346 used, and ChIs were identified by soma size and membrane properties (Chuhma et al., 2014). 
D2-EGFP single mutant mice were used for immunohistochemical evaluation of D2-EGFP expression in ChIs.

\section{Brain slice electrophysiology}

350 Mice were anesthetized with ketamine $(90 \mathrm{mg} / \mathrm{kg}) /$ xylazine $(7 \mathrm{mg} / \mathrm{kg})$. After confirmation of full

351 anesthesia, mice were decapitated and brains quickly removed in ice-cold high-glucose artificial

352 cerebrospinal fluid (ACSF) (in mM: $75 \mathrm{NaCl}, 2.5 \mathrm{KCl}, 26 \mathrm{NaHCO}_{3}, 1.25 \mathrm{NaH}_{2} \mathrm{PO}_{4}, 0.7 \mathrm{CaCl}_{2}, 2$

$353 \mathrm{MgCl}_{2}$ and 100 glucose, $\left.\mathrm{pH} 7.4\right)$ saturated with carbogen $\left(95 \% \mathrm{O}_{2}\right.$ and $\left.5 \% \mathrm{CO}_{2}\right)$. Coronal 300

$354 \mu \mathrm{m}$ Str sections were cut with a vibrating microtome (VT1200S, Leica, Buffalo Grove, IL),

355 allowed to recover in high glucose ACSF at room temperature for at least 1 hour, then

356 transferred to the recording chamber (submerged, $500 \mu 1$ volume) on the stage of an upright

357 microscope (BX61WI, Olympus, Tokyo, Japan), continuously perfused with standard ACSF (in

$358 \mathrm{mM}: 125 \mathrm{NaCl}, 2.5 \mathrm{KCl}, 25 \mathrm{NaHCO}_{3}, 1.25 \mathrm{NaH}_{2} \mathrm{PO}_{4}, 2 \mathrm{CaCl}_{2}, 1 \mathrm{MgCl}_{2}$ and 25 glucose, pH 7.4)

359 saturated with carbogen. ChR2-EYFP, D2-EGFP or ChAT-EGFP expression was confirmed by

$360470 \mathrm{~nm}$ LED field illumination; D1-tdTomato expression was confirmed by $530 \mathrm{~nm}$ LED

361 illumination (DC4100, Thorlabs, Newton, NJ). Recorded neurons were visualized using

362 enhanced visible light differential interference contrast (DIC) optics with a scientific c-MOS

363 camera (ORCA-Flash4.0LT, Hamamatsu Photonics, Hamamatsu, Japan).

364 In DAT ${ }^{\mathrm{IRES} c r e} ;$; $\mathrm{A} 32$ mice, ChIs were identified visually by large soma size, confirmed by

365 spontaneous firing, shallow resting membrane potentials (around $-60 \mathrm{mV}$ ) and voltage sag with -

366400 pA current injection (700 msec duration) (Chuhma et al., 2014). Recording patch pipettes (3-

$3677 \mathrm{M} \Omega$ ) were fabricated from standard-wall borosilicate glass capillary with filament (World

368 Precision Instruments). Intracellular solution for DA PSC recording was (in $\mathrm{mM}$ ): $135 \mathrm{~K}^{+}$- 
methane sulfonate $\left(\mathrm{MeSO}_{4}\right), 5 \mathrm{KCl}, 2 \mathrm{MgCl}_{2}, 0.1 \mathrm{CaCl}_{2}, 10 \mathrm{HEPES}, 1$ EGTA, 2 ATP and 0.1

371 better space clamp. For glutamate EPSC recording, $\mathrm{K}^{+}-\mathrm{MeSO}_{4}$ was replaced with $\mathrm{Cs}^{+}-\mathrm{MeSO}_{4}$, supplemented with QX314 (lidocaine N-ethyl bromide) $5 \mathrm{mM}$, to block action currents. For postrecording staining, Alexa Fluor 594 was added to the $\mathrm{Cs}^{+}-\mathrm{MeSO}_{4}$ based pipette solution. For $\mathrm{GABA}_{\mathrm{A}}$ IPSC recording, $\mathrm{K}^{+}-\mathrm{MeSO}_{4}$ was replaced with $\mathrm{CsCl}$ supplemented with QX314. All amplifier (Molecular Devices, San Jose, CA). Series resistance (8-30 M $\Omega$ ) was compensated illumination, through a 60x (NA 0.90) water-immersion objective (Olympus) for consistent activation of DA neuron terminals in the field of view.

DA neuron PSCs were pharmacologically isolated by blocking all other transmitter receptors, e.g. co-transmitted glutamate PSCs were isolated by blocking D1/5, D2, and GABA receptors.

385 The antagonist-isolation cocktail was applied at least $12 \mathrm{~min}$ prior to recording. Nicotinic and muscarinic ACh receptors were continuously blocked as well, since even in slices ChIs are spontaneously active and there is a basal cholinergic tone, that is likely to show regional heterogeneity. Antagonists and their concentrations were: iGluR AMPA/kainate CNQX $20 \mu \mathrm{M}$,

389 iGluR NMDA D-APV $50 \mu \mathrm{M}$, mGluR1 JNJ16259685 $5 \mu \mathrm{M}$, D1/5R SCH23390 $10 \mu \mathrm{M}, \mathrm{D} 2$ S(-)-

390 sulpiride $10 \mu \mathrm{M}, \mathrm{GABA}_{\mathrm{A}} \mathrm{R}$ SR95531 (gabazine) $10 \mu \mathrm{M}, \mathrm{GABA}_{\mathrm{B}} \mathrm{R}$ CGP55845 $3 \mu \mathrm{M}$, nAChR 391 mechamilamine $10 \mu \mathrm{M}$, mAChR scopolamine $2 \mu \mathrm{M}$. To achieve faster blockade of PSCs for 
392 confirmation of isolated responses (Extended Data Fig. 2), doubled concentration CNQX, D-

393 APV, or JNJ was used. Stock solutions of drugs were prepared in either water or DMSO, and

394 diluted by 1000 to 5000 fold in recording ACSF. Drugs were applied by perfusion. Locations of

395 recorded cells were mapped on mouse atlas coronal sections (Paxinos and Franklin, 2008), on a

$39650 \mu \mathrm{m}$ medial-lateral and dorsal-ventral grid.

397 Voltage clamp data were filtered at $5 \mathrm{kHz}$ with a 4-pole Bessel filter, digitized at $5 \mathrm{kHz}$ (Digidata

398 1550A, Molecular Devices), recorded using pClamp 10 (Molecular Devices;

399 RRID:SCR_011323), and analysed with Axograph X (Axograph Science; RRID:SCR_014284).

400 PSCs were measured from averages of 10 consecutive traces. Synaptic responses were measured

401 starting with the second stimulus to avoid artificially large responses to the first stimulus elicited

402 after a period of rest. Because of synchronized and fast responses, iGluR and GABA $\mathrm{PSCs}$ were

403 evaluated by peak amplitude measured in a $15 \mathrm{msec}$ post-stimulus window. mGluR, D2R, and

404 D1/5R PSCs in ChIs were evaluated by charge transfer measured in a $1.5 \mathrm{sec}$ post-stimulus

405 window, as area under the curve. D1/5R PSCs in FSIs were evaluated in a 0.3 sec window due to

406 their shorter time course (slower than PSCs in ChIs), and because they were not as synchronized

407 (as glutamate or $\mathrm{GABA}_{\mathrm{A}} \mathrm{R}$ PSCs). GABA $\mathrm{ASC}$ declined quickly with the first 10 stimuli

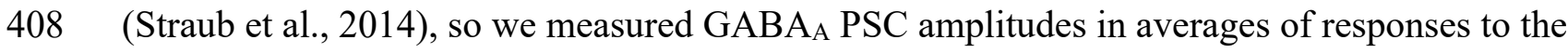

409 second 10 stimuli. Rise time (10-90\% peak) and width at 50\% peak of D1/5R PSCs were

410 measured in ChIs with responses greater than $2 \mathrm{pC}$ and FSIs with peak amplitudes greater than 8

411 pA. Baseline charge transfer was measured from 10 consecutive traces

412 without photostimulation. For comparison between ChIs identified by

413 ChAT-EGFP fluorescence and ChIs identified by post-recording

414 staining, we chose three discrete locations and compared PSC 
measures. The three locations were NAc medial shell (in NAc shell

region in mouse atlas, and $\mathrm{ML} \leq+1$; in $\mathrm{mm}$ from bregma ), medial

417 caudate putamen ( $\mathrm{CPu})(\mathrm{AP} \geq+0.26$ and $\leq+1.1, \mathrm{ML} \geq+2.1$, DV $\geq-3.8)$, and

418 lateral $\mathrm{CPu}(\mathrm{AP} \geq+0.95$ and $\leq+1.34, \mathrm{ML} \leq+1.15, \mathrm{DV} \geq-3.7)$. In total 1677

419 cells were used for this study, and among them, 34 were the same cells

420 reported in Chuhma et al. (2018). A maximum of 10 cells were recorded

421 per animal.

\section{Immunocytochemistry}

423 Three D2-EGFP mice (P74, 1 male and 2 females) were anesthetized with ketamine/xylazine and

424 perfused with cold phosphate buffered saline (PBS) followed by $4 \%$ paraformaldehyde (PFA).

425 Brains were removed and post-fixed overnight in 4\% PFA. Coronal $50 \mu \mathrm{m}$ sections were cut

426 using a vibrating microtome (Leica VT1200S), and stored in a cryoprotectant solution (30\%

427 glycerol, $30 \%$ ethylene glycol in $0.1 \mathrm{M}$ Tris $\mathrm{HCl} \mathrm{pH} \mathrm{7.4)} \mathrm{at}-20^{\circ} \mathrm{C}$ until processing. Sections

428 were washed in PBS and incubated in glycine $(100 \mathrm{mM})$ for $30 \mathrm{~min}$ to quench aldehydes. Non-

429 specific binding was blocked with 10\% normal goat serum (NGS; Millipore, St. Louis, MO) in

$430 \quad 0.1$ PBS Triton X-100 (PBS-T) for 2 hours. Primary antibodies in $0.02 \%$ PBS-T and $2 \%$ NGS

431 were applied for 24 hours, at $4{ }^{\circ} \mathrm{C}$ on a shaker. Primary antibodies were: anti-EGFP $(1: 2,000$

432 dilution, rabbit polyclonal, Millipore, RRID:AB_91337) and anti-choline acetyltransferase

433 (ChAT; 1:1000 dilution, goat polyclonal, Millipore, RRID:AB_2079751). For post-recording

434 staining, slices were fixed with 4\% PFA at room temperature for 1-3 hours. After washing with

435 PBS, slices were processed as above, except that the primary antibody (anti ChAT) was applied

436 for 2-3 days. The recorded cells were observed directly with Alexa Fluor 594 dye fluorescence 
437 without immunostaining. Sections were then washed with PBS and secondary antibodies applied

438 for $45 \mathrm{~min}$ in $0.02 \%$ PBS-T at room temperature. Secondary antibodies (1:200 dilution;

439 ThermoFisher Scientific, Waltham, MA) were: anti-goat Alexa Fluor 488 (RRID:AB_2534102),

440 anti-goat Alexa Fluor 594 (RRID:AB_2534105) and anti-rabbit Alexa Fluor 488

441 (RRID:AB_2535792). Sections were mounted on gelatin subbed slides (Southern Biotech,

442 Birmingham, AL) and cover slipped with Prolong Gold aqueous medium (ThermoFisher

443 Scientific) and stored at $4{ }^{\circ} \mathrm{C}$.

\section{$444 \quad$ Imaging and cell counting}

445 Images were acquired with an AxioImager.M2 fluorescence microscope and ZEN Digital

446 Imaging software (Zeiss; RRID:SCR_013672) using a 20x objective for GFP/ChAT

447 colocalization and a 40x objective for Alexa 594/ChAT colocalization and dendrite tracing.

448 Images were taken in $1 \mu \mathrm{m}$ steps subtending the entire slice thickness (26-37 images per slice),

449 and each z-section image was examined for immuno- or dye-fluorescence. $\mathrm{ChAT}^{+}$and

$450 \mathrm{ChAT}^{+} / \mathrm{GFP}^{+}$cells were counted using the Optical Fractionator Probe in Stereo Investigator 11

451 (MBF Bioscience, Williston, VT; RRID:SCR_002526) using a 10x objective, over 20\% of the

452 area of every 5th slice. In each brain, 12-15 slices were analyzed. Stereological studies were

453 performed unilaterally.

454 EGFP fluorescence intensity and soma size measurements in D2-EGFP mouse slices were

455 measured with Image J (FIJI) 2.0.0 (NIH, RRID:SCR_003070). Regions of interest (ROIs) were

456 made by manual tracing of $\mathrm{ChAT}^{+}$or $\mathrm{GFP}^{+}$somas, and the area used as a measure of soma size.

457 Average GFP staining intensity in each ROI was regarded as GFP intensity of each cell. For 
$458 \mathrm{ChAT}^{+} / \mathrm{GFP}^{+}$cells, ROIs made in ChAT channel projected to the GFP channel and measured 459 averaged GFP staining intensity within those.

460 Dendrites of Alexa-filled ChIs were traced with Image J 2.0.0 using Simple Neurite Tracer plug-

461 in. The same set of cells used for post-recording ChAT staining were used for dendrite tracing,

462 excluding one cell with faint dendrite staining. Tracing was done in Alexa 594 z-stack images.

463 For visualization of fine dendrites, contrast was enhanced. Dendrites were semi-automatically

464 traced until dendrites became not recognizable or reached a cut end. Generally, tracing extended

465 to the tertiary dendrites. Lengths of all measured dendrites of individual cells were summed.

\section{Statistics for electrophysiology and immunostaining}

467 Statistics, including sample size estimation, were done in R3.6.1 (RRID:SCR_001905) with

468 RStudio 1.2.1335 (RRID:SCR_000432). The following packages were used in addition to

469 base/stats; pwr 1.2-2 (for sample size estimation), WebPower 0.5.2 (for sample size estimation),

470 afex 0.25-1 (for Type III sum of square ANOVA), emmeans 1.4.1 (for post-hoc comparison).

471 We used parametric tests, as the variables we measured were continuous numeric variables (not

472 ranked variables).

473 Sample sizes were estimated with power $=0.8$ and alpha $=0.05$. Effect sizes were calculated and

474 estimated from previously performed similar experiments. For paired t-test (antagonist effects),

475 Cohen's d was set to 1.6 (very strong effect), suggesting 6 cells (pairs) were required. For one-

476 way ANOVA for image analysis, Cohen's f was set to 0.5 (strong effect), suggesting that a total

47742 cells, in 3 groups. For two-way ANOVA, Cohen's f was set to 0.6 based on a previously

478 performed regional heterogeneity study, giving a sample size of 30 cells, in 6 groups. For the 
correlation study, a significance criterion of $\mathrm{R}^{2}=0.5(\mathrm{R}=0.71)$ gave 13 as the required number of observations.

Data are presented as mean \pm S.E.M, unless otherwise noted. Kormogorov-Smirnov tests were one-tailed, while all other tests were two-tailed. The test used, $\mathrm{t}$ value, $\mathrm{F}$ value, $\mathrm{R}^{2}$, degree of freedom, $\mathrm{p}$ value, or exact number of samples (n), and what $\mathrm{n}$ stands for are reported in either the main text or figure legends. For antagonist effects on pharmacologically isolated PSCs (Fig. 2), we include a separate table with statistical results (Table S1). Significance was set at $\mathrm{p}<0.05$.

\section{Spatial data analysis}

All spatial data analyses were done in R3.6.1. The following packages were used for data analysis and plotting, in addition base/stats; dplyr 0.8.1 (RRID:SCR_016708), tidyr 0.8.3 (RRID:SCR_003005), purrr 0.3.2, readr 1.3.1, readx1 1.3.1, ggplot2 3.1.1 (RRID:SCR_014601),

RColorBrewer 1.1-2 (RRID:SCR_016697), gridExtra 2.3, egg 0.4.2, cowplot 0.9.4, plotly 4.9.0 (RRID:SCR_013991), sp 1.3-1, gstat 2.0-2, factoextra 1.0.5 (RRID:SCR_016692), and ClustGeo 2.0 .

PSC sizes were estimated on a $150 \mu \mathrm{m}$ grid, manually overlaid on coronal sections $(\sim 150 \mu \mathrm{m}$ apart) of a mouse brain atlas (Paxinos and Franklin, 2008). In the unilateral Str, the number of grid lattices was 4177 . Estimation was done by ordinary block kriging with a $450 \mu \mathrm{m}$ cube block with gstat 2.0-2 (Gräler et al., 2016). Experimental variograms were fit with Matern covariance functions, and parameters (psill, nugget, and range) of each map are reported in Table S2.

498 Hierarchical clustering of kriging results was done with spatial constraint with ClustGeo 2.0

499 (Chavent et al., 2018). Estimated PSC sizes were normalized by the maximum and minimum 500 values of each map before clustering. Optimal numbers of clusters $(\mathrm{K})$ were chosen by the 
501 silhouette method with factoextra 1.0.5 in 3-10 clusters to obtain contiguous subdomains. After

502 an optimal K was obtained, mean and SD of PSCs in each cluster were calculated, and compared

503 to the results among $\mathrm{K}$ and $\mathrm{K} \pm 1$ clusters, to confirm that the $\mathrm{K}$ was optimal. If there were more

504 than two clusters with almost the same set of mean values, K was likely to be too large (too

505 many clusters). If adding one more cluster largely reduced SD in clusters, K was likely too small.

506 The chosen $\mathrm{K}$ was optimal in cell-type clusters, while $\mathrm{K}+1$ was optimal for transmitter clusters.

507 Location and data (PSC size) dissimilarity matrices were calculated using the Euclidean

508 distances of values between data points.

509 To obtain the optimal mixing parameter alpha, a quality criterion (Q) was calculated in 0.1 steps

510 with the ClustGeo 2.0 choicealpha function (Chavent et al., 2018). Alpha ranges from 0, where

511 clustering is determined solely by location, to 1 , where clustering is determined solely by data

512 (PSC size). With increasing alpha, the data matrix contribution is reduced, while the location

513 matrix contribution is increased. Generally, reduction of the data matrix-contribution caused

514 reduced homogeneity of data in the clusters (reducing data Q), while geographic (location)

515 coherence increased (increasing location Q). Alpha is optimized when location Q increases

516 significantly, while minimizing data Q reduction. However, our datasets were distributed in three

517 dimensions and we needed stronger location constraint than the ward-wise socio-demographic

518 analysis for which the ClustGeo package was developed (Chavent et al., 2018), to obtain

519 contiguous subdomains. Therefore, we used the alpha value just after location and data

520 normalized Q plot curves crossed. The determined alphas were: dSPN 0.3, iSPN 0.3, ChI 0.4,

521 FSI 0.4, glutamate 0.3, DA 0.4, and GABA 0.3. After clustering with the determined alpha,

522 dendrograms were cut at the points to obtain optimal number of clusters. Means and SDs of

523 transmitter receptor/cell type responses in each cluster were calculated. We used the number of 
524 grid points in each cluster to estimate the volume of each cluster. To evaluate the quality of

525 clustering, $\mathrm{F}$ values were calculated for each variable. $\mathrm{F}$ is defined as between-cluster variance

526 divided by within-cluster variance, and magnitudes of $\mathrm{F}$ values indicate how well the respective

527 variables discriminate between clusters (Calinski and Harabasz, 1974). 
bioRxiv preprint doi: https://doi.org/10.1101/2020.10.11.334961; this version posted October 11,2020. The copyright holder for this preprint

(which was not certified by peer review) is the author/funder, who has granted bioRxiv a license to display the preprint in perpetuity. It is made available under aCC-BY-NC-ND 4.0 International license.

\section{Acknowledgements}

529 We thank Casey Brody and Sarah Garcia for technical assistance. This work was supported by

530 NIH R01 DA038966 and R01 MH117128 (SR).

\section{Competing interests}

532 Authors declare no competing interests. 


\section{References}

535 Bäckman, C.M., Malik, N., Zhang, Y., Shan, L., Grinberg, A., Hoffer, B.J., Westphal, H., and

536 Tomac, A.C. (2006). Characterization of a mouse strain expressing Cre recombinase from the 3'

537 untranslated region of the dopamine transporter locus. Genesis 44, 383-390.

538 Beckstead, M.J., Grandy, D.K., Wickman, K., and Williams, J.T. (2004). Vesicular dopamine

539 release elicits an inhibitory postsynaptic current in midbrain dopamine neurons. Neuron 42, 939-

540946.

541 Beeler, J.A., and Kisbye Dreyer, J. (2019). Synchronicity: The Role of Midbrain Dopamine in

542 Whole-Brain Coordination. eNeuro 6, e0345.

543 Bivand, R., Pebesma, E.J., and Gómez-Rubio, V. (2013). Applied spatial data analysis with R,

544 Second edition edn (New York: Springer).

545 Cai, L.X., Pizano, K., Gundersen, G.W., Hayes, C.L., Fleming, W.T., Holt, S., Cox, J.M., and

546 Witten, I.B. (2020). Distinct signals in medial and lateral VTA dopamine neurons modulate fear

547 extinction at different times. eLIFE 9, e54936.

548 Cai, Y., and Ford, C.P. (2018). Dopamine cells differentially regulate striatal cholinergic

549 transmission across regions through corelease of dopamine and glutamate. Cell Rep 25, 3148-

5503157.

551 Calinski, T., and Harabasz, J. (1974). A dendrite method for cluster analysis. Communications in

552 Statistics - Theory and Methods 3, 1-27. 
553 Centonze, D., Grande, C., Usiello, A., Gubellini, P., Erbs, E., Martin, A.B., Pisani, A., Tognazzi,

554 N., Bernardi, G., Moratalla, R., Borrelli, E., and Calabresi, P. (2003). Receptor subtypes

555 involved in the presynaptic and postsynaptic actions of dopamine on striatal interneurons. $\mathrm{J}$

556 Neurosci 23, 6245-6254.

557 Chavent, M., Kuentz-Simonet, V., Labenne, A., and Saracco, J. (2018). ClustGeo: an R package

558 for hierarchical clustering with spatial constraints. Computational Statistics 33, 1799-1822.

559 Chuhma, N., Mingote, S., Moore, H., and Rayport, S. (2014). Dopamine neurons control striatal

560 cholinergic neurons via regionally heterogeneous dopamine and glutamate signaling. Neuron 81 ,

$561901-912$.

562 Chuhma, N., Mingote, S., Yetnikoff, L., Kalmbach, A., Ma, T., Ztaou, S., Sienna, A.C., Tepler,

563 S., Poulin, J.F., Ansorge, M., Awatramani, R., Kang, U.J., and Rayport, S. (2018). Dopamine

564 neuron glutamate cotransmission evokes a delayed excitation in lateral dorsal striatal cholinergic

565 interneurons. eLIFE 7, e39786.

566 Chuhma, N., Zhang, H., Masson, J., Zhuang, X., Sulzer, D., Hen, R., and Rayport, S. (2004).

567 Dopamine neurons mediate a fast excitatory signal via their glutamatergic synapses. J Neurosci

$568 \quad 24,972-981$.

569 Crittenden, J.R., Lacey, C.J., Lee, T., Bowden, H.A., and Graybiel, A.M. (2014). Severe drug-

570 induced repetitive behaviors and striatal overexpression of VAChT in ChAT-ChR2-EYFP BAC

571 transgenic mice. Frontiers in Neural Circuits 8, 57. 
572 Dal Bo, G., St-Gelais, F., Danik, M., Williams, S., Cotton, M., and Trudeau, L.-E. (2004).

573 Dopamine neurons in culture express VGLUT2 explaining their capacity to release glutamate at 574 synapses in addition to dopamine. J Neurochem 88, 1398-1405.

575 Gerfen, C.R., and Surmeier, D.J. (2011). Modulation of striatal projection systems by dopamine.

576 Annu Rev Neurosci 34, 441-466.

577 Gräler, B., Pebesma, E., and Heuvelink, G. (2016). Spatio-Temporal Interpolation using gstat.

578 The R Journal 8, 204.

579 Haber, S.N., Fudge, J.L., and McFarland, N.R. (2000). Striatonigrostriatal pathways in primates

580 form an ascending spiral from the shell to the dorsolateral striatum. J Neurosci 20, 2369-2382.

581 Heymann, G., Jo, Y.S., Reichard, K.L., McFarland, N., Chavkin, C., Palmiter, R.D., Soden,

582 M.E., and Zweifel, L.S. (2020). Synergy of distinct dopamine projection populations in

583 behavioral reinforcement. Neuron 105, 909-920.

584 Hnasko, T.S., Chuhma, N., Zhang, H., Goh, G.Y., Sulzer, D., Palmiter, R.D., Rayport, S., and

585 Edwards, R.H. (2010). Vesicular glutamate transport promotes dopamine storage and glutamate

586 corelease in vivo. Neuron 65, 643-656.

587 Ikemoto, S. (2007). Dopamine reward circuitry: two projection systems from the ventral

588 midbrain to the nucleus accumbens-olfactory tubercle complex. Brain Res Rev 56, 27-78.

589 Kawaguchi, Y. (1993). Physiological, morphological, and histochemical characterization of three

590 classes of interneurons in rat neostriatum. J Neurosci 13, 4908-4923. 
591 Kim, J.-I., Ganesan, S., Luo, S.X., Wu, Y.-W., Park, E., Huang, E.J., Chen, L., and Ding, J.B.

592 (2015). Aldehyde dehydrogenase la1 mediates a GABA synthesis pathway in midbrain

593 dopaminergic neurons. Science (New York, NY) 350, 102-106.

594 Kramer, D.J., Risso, D., Kosillo, P., Ngai, J., and Bateup, H.S. (2018). Combinatorial expression

595 of Grp and Neurod6 defines dopamine neuron populations with distinct projection patterns and

596 disease vulnerability. eNeuro 5, e0152.

597 Kreitzer, A.C. (2009). Physiology and pharmacology of striatal neurons. Annu Rev Neurosci 32,

$598 \quad 127-147$.

599 Lacey, C.J., Boyes, J., Gerlach, O., Chen, L., Magill, P.J., and Bolam, J.P. (2005). GABA(B)

600 receptors at glutamatergic synapses in the rat striatum. Neuroscience 136, 1083-1095.

601 Lim, S.A., Kang, U.J., and McGehee, D.S. (2014). Striatal cholinergic interneuron regulation and 602 circuit effects. Front Synaptic Neurosci 6, 22.

603 Lin, R., Liang, J., Wang, R., Yan, T., Zhou, Y., Liu, Y., Feng, Q., Sun, F., Li, Y., Li, A., Gong, 604 H., and Luo, M. (2020). The Raphe Dopamine System Controls the Expression of Incentive 605 Memory. Neuron 106, 498-514.

606 Liu, C., and Kaeser, P.S. (2019). Mechanisms and regulation of dopamine release. Curr Opin 607 Neurobiol 57, 46-53.

608 Marcott, P.F., Mamaligas, A.A., and Ford, C.P. (2014). Phasic dopamine release drives rapid 609 activation of striatal D2-receptors. Neuron 84, 164-176.

610 Matheron, G. (1963). Principles of geostatistics. Economic Geology 58, 1246-1266. 
611 Mingote, S., Amsellem, A., Kempf, A., Rayport, S., and Chuhma, N. (2019). Dopamine-

612 glutamate neuron projections to the nucleus accumbens medial shell and behavioral switching.

$613 \quad$ Neurochem Int 129, 104482.

614 Morales, M., and Margolis, E.B. (2017). Ventral tegmental area: cellular heterogeneity,

615 connectivity and behaviour. Nat Rev Neurosci 18, 73-85.

616 Nagy, P.M., and Aubert, I. (2012). Overexpression of the vesicular acetylcholine transporter

617 increased acetylcholine release in the hippocampus. Neuroscience 218, 1-11.

618 Parker, N.F., Cameron, C.M., Taliaferro, J.P., Lee, J., Choi, J.Y., Davidson, T.J., Daw, N.D., and

619 Witten, I.B. (2016). Reward and choice encoding in terminals of midbrain dopamine neurons

620 depends on striatal target. Nat Neurosci 19, 845-854.

621 Paxinos, G., and Franklin, K.B.G. (2008). The Mouse Brain in Stereotaxic Coordinates, 3, 622 illustrated edn (Amsterdam; Boston: Academic Press).

623 Poulin, J.F., Caronia, G., Hofer, C., Cui, Q., Helm, B., Ramakrishnan, C., Chan, C.S., Dombeck,

624 D.A., Deisseroth, K., and Awatramani, R. (2018). Mapping projections of molecularly defined

625 dopamine neuron subtypes using intersectional genetic approaches. Nat Neurosci 21, 1260-1271.

626 Rousseeuw, P.J. (1987). Silhouettes: A graphical aid to the interpretation and validation of

627 cluster analysis. Journal of Computational and Applied Mathematics 20, 53-65.

628 Shi, W.-X., and Rayport, S. (1994). GABA synapses formed in vitro by local axon collaterals of 629 nucleus accumbens neurons. J Neurosci 14, 4548-4560. 
630 Straub, C., Tritsch, N.X., Hagan, N.A., Gu, C., and Sabatini, B.L. (2014). Multiphasic

631 modulation of cholinergic interneurons by nigrostriatal afferents. J Neurosci 34, 8557-8569.

632 Stuber, G.D., Hnasko, T.S., Britt, J.P., Edwards, R.H., and Bonci, A. (2010). Dopaminergic

633 terminals in the nucleus accumbens but not the dorsal striatum corelease glutamate. J Neurosci

$63430,8229-8233$.

635 Sulzer, D., Cragg, S.J., and Rice, M.E. (2016). Striatal dopamine neurotransmission: regulation

636 of release and uptake. Basal ganglia 6, 123-148.

637 Sulzer, D., Joyce, M.P., Lin, L., Geldwert, D., Haber, S.N., Hattori, T., and Rayport, S. (1998).

638 Dopamine neurons make glutamatergic synapses in vitro. J Neurosci 18, 4588-4602.

639 Surmeier, D.J., Carrillo-Reid, L., and Bargas, J. (2011). Dopaminergic modulation of striatal

640 neurons, circuits, and assemblies. Neuroscience 198, 3-18.

641 Tecuapetla, F., Patel, J.C., Xenias, H., English, D., Tadros, I., Shah, F., Berlin, J., Deisseroth, K.,

642 Rice, M.E., Tepper, J.M., and Koos, T. (2010). Glutamatergic signaling by mesolimbic

643 dopamine neurons in the nucleus accumbens. J Neurosci 30, 7105-7110.

644 Tepper, J.M., Koós, T., Ibanez-Sandoval, O., Tecuapetla, F., Faust, T.W., and Assous, M.

645 (2018). Heterogeneity and diversity of striatal GABAergic interneurons: Update 2018. Front

646 Neuroanat 12, 91.

647 Tritsch, N.X., Ding, J.B., and Sabatini, B.L. (2012). Dopaminergic neurons inhibit striatal output

648 through non-canonical release of GABA. Nature 490, 262-266. 
649 Tritsch, N.X., Oh, W.-J., Gu, C., and Sabatini, B.L. (2014). Midbrain dopamine neurons sustain

650 inhibitory transmission using plasma membrane uptake of GABA, not synthesis. eLIFE 3,

$651 \mathrm{e} 01936$.

652 Wieland, S., Du, D., Oswald, M.J., Parlato, R., Kohr, G., and Kelsch, W. (2014). Phasic

653 dopaminergic activity exerts fast control of cholinergic interneuron firing via sequential NMDA,

654 D2, and D1 receptor activation. J Neurosci 34, 11549-11559.

655 Wu, J., Kung, J., Dong, J., Chang, L., Xie, C., Habib, A., Hawes, S., Yang, N., Chen, V., Liu, Z.,

656 Evans, R., Liang, B., Sun, L., Ding, J., Yu, J., Saez-Atienzar, S., Tang, B., Khaliq, Z., Lin, D.T.,

657 Le, W., and Cai, H. (2019). Distinct connectivity and functionality of aldehyde dehydrogenase

658 1a1-positive nigrostriatal dopaminergic neurons in motor learning. Cell Rep 28, 1167-1181. 


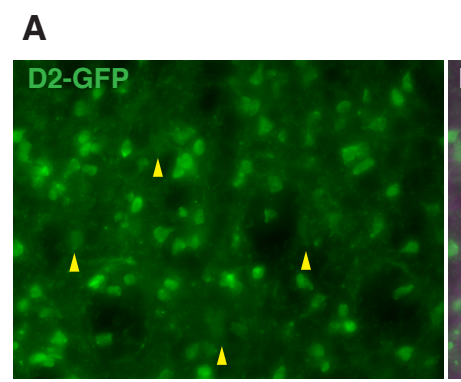

B ChAT ${ }^{+}$neurons in D2-EGFP mice

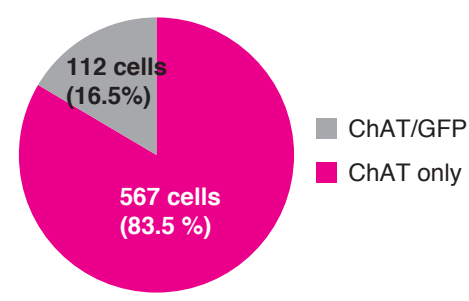

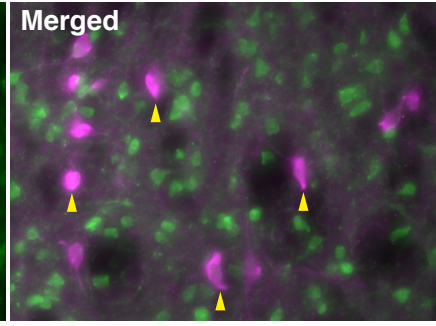

C

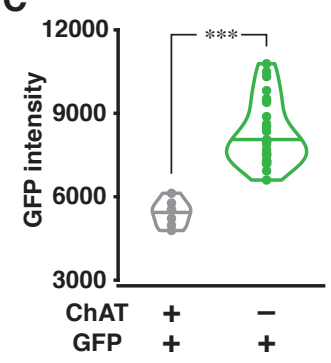

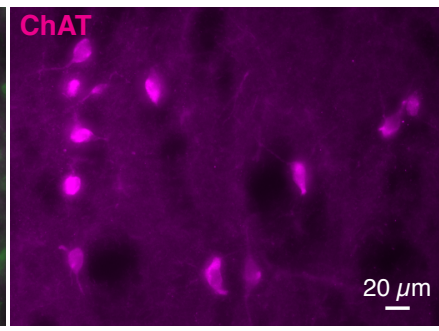

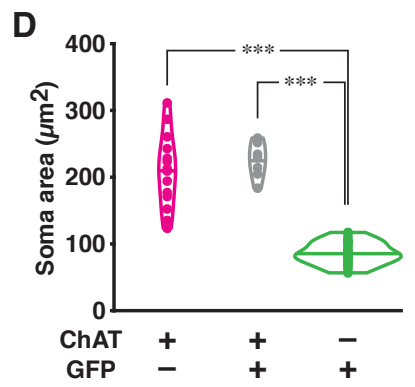

\section{Supplementary Fig 1}

\section{EGFP expression in D2-EGFP mice.}

In slices from D2-EGFP mice, brighter $\mathrm{EGFP}^{+}$neurons were iSPNs and not ChIs. (A) Representative EGFP (green) and ChAT (magenta) immunostaining in the Str of a D2-EGFP mouse. Yellow arrows indicate $\mathrm{EGFP}^{+} / \mathrm{ChAT}^{+}$cells. (B) The ratio of $\mathrm{EGFP}^{+}$(gray) and $\mathrm{EGFP}^{-}$(magenta) $\mathrm{ChAT}^{+}$neurons. Cell numbers are stereological counts from three mice. (C) EGFP fluorescence intensity in $\mathrm{ChAT}^{+}$(gray) and $\mathrm{ChAT}^{-}$ (green) neurons. EGFP fluorescence in $\mathrm{EGFP}^{+} / \mathrm{ChAT}^{+}$neurons was dimmer than in $\mathrm{EGFP}^{+} / \mathrm{ChAT}^{-}$neurons. Each dot shows the average EGFP fluorescence intensity of a single soma. Outlines in the graph show densities of counts and horizontal lines in the outlines show medians of the intensities. $\mathrm{EGFP}^{+} / \mathrm{ChAT}^{+} \mathrm{n}=7$ cells, $\mathrm{EGFP}^{+} / \mathrm{ChAT}^{-} \mathrm{n}$ $=28$ cells, Welch's t-test, $\mathrm{t}=-10.3, \mathrm{df}=25.2, \mathrm{p}<0.0001$. (D) Soma size analysis of $\mathrm{EGFP}^{-} / \mathrm{ChAT}^{+}$(magenta), $\mathrm{EGFP}^{+} / \mathrm{ChAT}^{+}$(gray), and $\mathrm{EGFP}^{+} / \mathrm{ChAT}^{-}$(green) neurons. $\mathrm{ChAT}^{+}$neurons, regardless of EGFP expression, were significantly larger than $\mathrm{ChAT}^{-}$ neurons. $\mathrm{EGFP}^{-} / \mathrm{ChAT}^{+} \mathrm{n}=14$ cells, one-way ANOVA, $\mathrm{F}_{(2,46)}=88.8, \mathrm{p}<0.0001$. Pairwise comparison as post-hoc test (with Bonferroni correction) $\mathrm{EGFP}^{-} / \mathrm{ChAT}^{+}-$ $\mathrm{EGFP}^{+} / \mathrm{ChAT}^{+}, \mathrm{p}=0.92 ; \mathrm{EGFP}^{-} / \mathrm{ChAT}^{+}-\mathrm{EGFP}^{+} / \mathrm{ChAT}^{-}, \mathrm{p}<0.0001 ; \mathrm{EGFP}^{+} / \mathrm{ChAT}^{+}$ $-\mathrm{EGFP}^{+} / \mathrm{ChAT}^{-}, \mathrm{p}<0.0001$. 


\section{A}

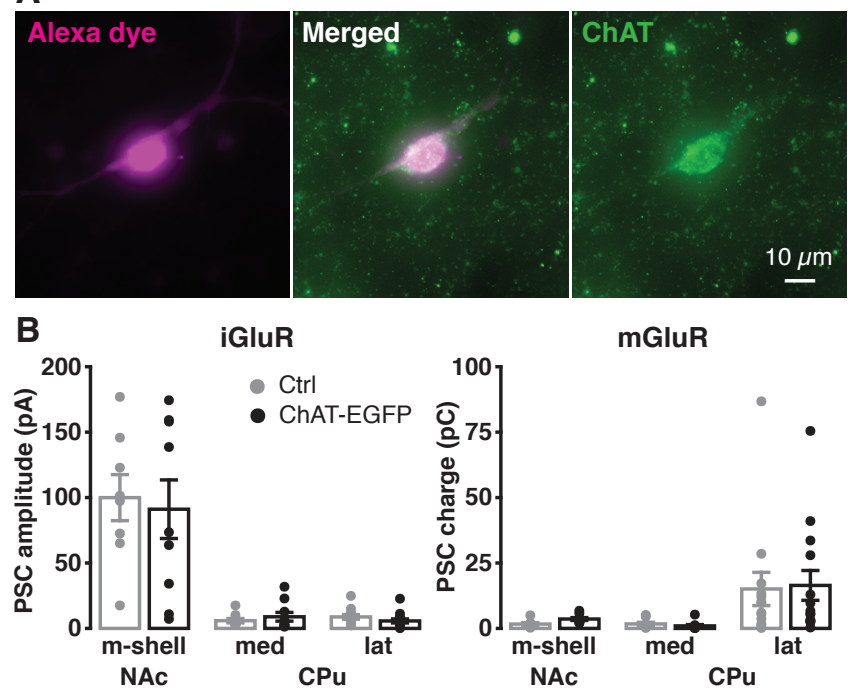

\section{Supplemental Fig 2}

Glutamate responses in ChAT-EGFP mice and mice without ChAT-EGFP.

VAChT overexpression in ChAT-EGFP mice did not affect measures of regional heterogeneity. The identification of ChIs in mice without ChAT-EGFP (DAT ${ }^{\text {IREScre/+; }}$; ChR2) was confirmed by post-recording ChAT immunostaining. (A) Images of a recorded ChI filled with Alexa Fluor 594 (magenta), ChAT counter staining of the cells (green), and a merged image (white). Note that the green channel also includes DA neuron terminals with ChR2-EYFP fluorescence. (B) iGluR (left) and mGluR (right) PSCs sizes on ChIs in NAc medial shell (m-shell), medial caudate-putamen (med $\mathrm{CPu}$ ), and lateral (lat) $\mathrm{CPu}$ from mice without ChAT-EGFP (Ctrl; gray) and with ChAT-EGFP (black). There were no genotypic differences. Recorded number of cells were: NAc m-shell Ctrl $n=8$ cells, ChAT-EGFP $n=9$ cells; med CPu Ctrl $n$ $=9$ cells, ChAT-EGFP $n=10$ cells; lat $\mathrm{CPu}$ Ctrl $\mathrm{n}=13$ cells, ChAT-EGFP $\mathrm{n}=14$ cells. iGluR: location $\mathrm{F}_{(2,57)}=49.5, \mathrm{p}<0.0001$; genotype $\mathrm{F}_{(1,57)}=0.14, \mathrm{p}=0.71$; interaction $\mathrm{F}_{(2,57)}=0.16, \mathrm{p}=0.85$; two-way ANOVA. mGluR: location $\mathrm{F}_{(2,57)}=6.8, \mathrm{p}$ $=0.002$, genotype $F_{(1,57)}=0.05, p=0.82$, interaction $F_{(2,57)}=0.04, p=0.96$; two-way ANOVA. 


\section{Fig S3}

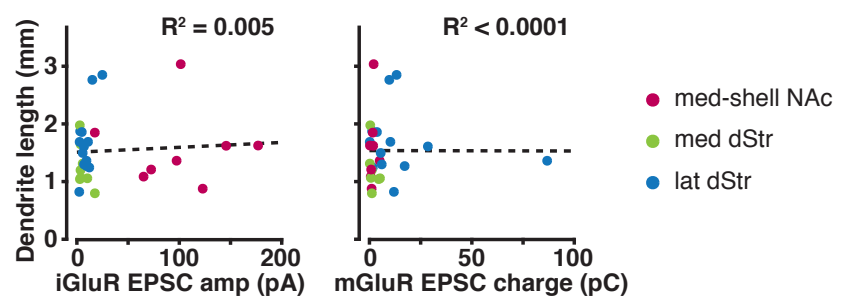

\section{Supplemental Fig 3}

\section{Correlation between dendrite lengths and PSC sizes.}

Regional differences in synaptic responses were not due to regional differences in the truncation of dendrites by slicing. The same set of cells used in Supplemental Fig 2 were analyzed. One cell did not show good dendrite staining and was discarded. Dendrite tracing was done in Alexa Fluor images with contrast enhancement. Total lengths of traceable dendrites (in $\mathrm{mm}$ ) were plotted against amplitude of iGluR PSCs (left) or charge transfer of mGluR PSCs (right). Locations of recorded cells are color coded: NAc m-shell, dark red; med $\mathrm{CPu}$, light green; lat $\mathrm{CPu}$, blue. Smaller responses did not correlate with shorter dendrites. iGluR: $\mathrm{R}^{2}=0.005, \mathrm{~F}_{(1,27)}=0.145, \mathrm{p}=0.71$. mGluR: $\mathrm{R}^{2}<0.0001, \mathrm{~F}_{(1,27)}=0.0001, \mathrm{p}=0.99$. 


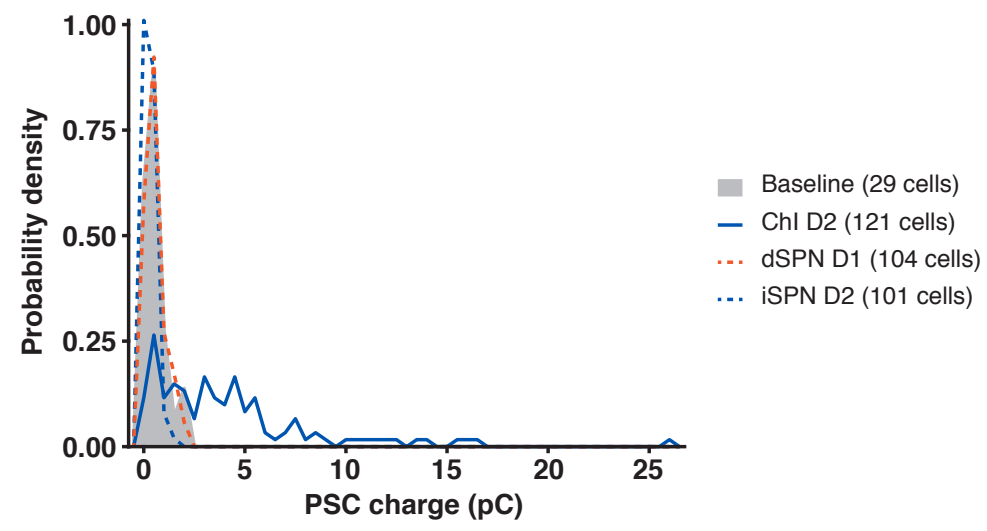

\section{Supplemental Fig 4}

No significant DA responses in dSPNs or iSPNs.

D1/5R PSC charge transfer in dSPNs (orange broken line) and D2R PSC charge transfer in iSPNs (blue broken line) were compared to baseline (gray shading). For baseline, charge transfers were measured in a $1.5 \mathrm{sec}$ time window without photostimulation. The charge transfer histograms (bin width $0.5 \mathrm{pC}$ ) were expressed as a probability density, and outlines of the histograms are shown. For comparison, the probability density curve of the positive response of D2R PSCs in ChI is shown (blue solid line). Comparison to baseline ( $\mathrm{n}=29$ cells): D1/5R PSC in dSPN, $\mathrm{n}=$ 104 cells, $\mathrm{D}=0.13, \mathrm{p}=0.48$; D2R PSC in iSPN, $\mathrm{n}=101$ cells, $\mathrm{D}=0.14$, $\mathrm{p}=0.42 ; \mathrm{D} 2 \mathrm{R}$ PSC in ChI, $\mathrm{n}=121$ cells, $\mathrm{D}=0.65, \mathrm{p}<0.0001$ one-tailed two-sample Kolmogorov-Smirnov test. 


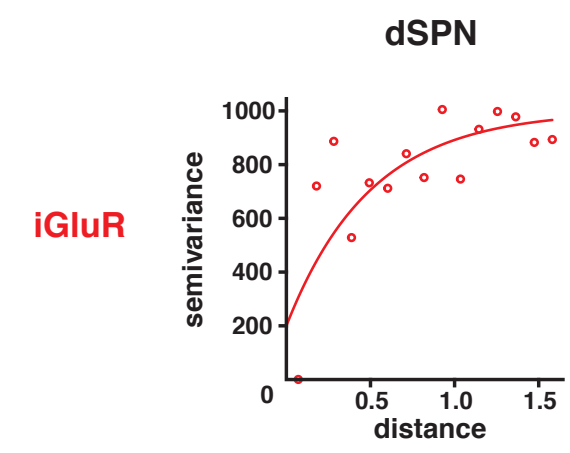

mGluR

D2R

D1/5R

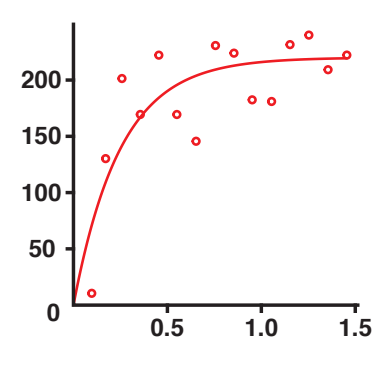

Chl

FSI
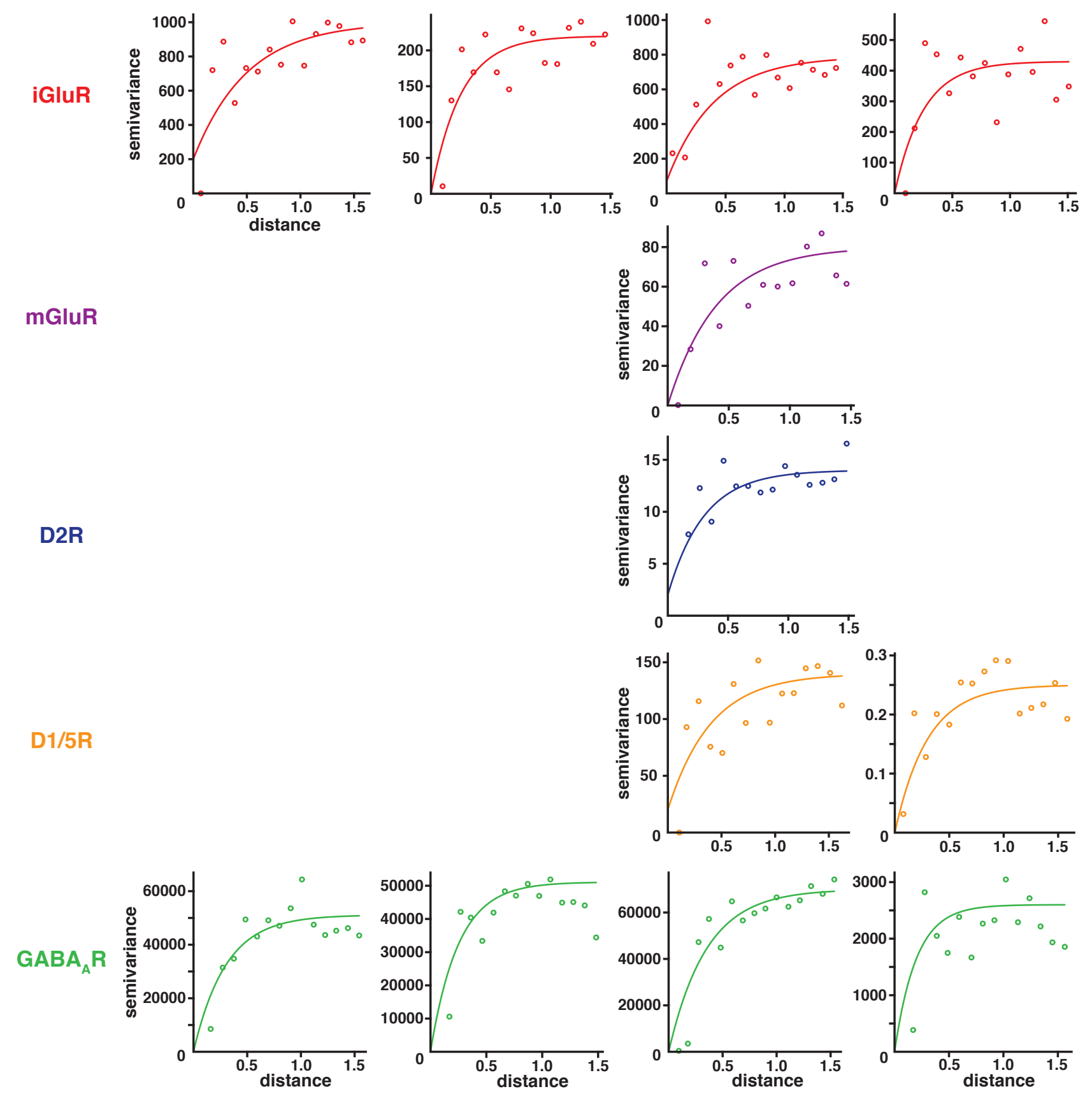

\section{Supplemental Fig 5}

Experimental variograms of recorded PSCs.

Semivariance of PSCs in pairs of recording locations (vertical axis) were plotted versus euclidean distance of these locations (in $\mathrm{mm}$ ). Averages in $0.1 \mathrm{~mm}$ distance bins are shown as open circles in the graphs. Variograms were fit by Matern covariance functions (lines), and the fitted values were used to obtain weights for averaging to estimate PSC sizes on a cubic grid. See Supplemental Table 2 for fitting parameters. 
bioRxiv preprint doi: https://doi.org/10.1101/2020.10.11.334961; this version posted October 11,2020. The copyright holder for this preprint (which was not certified by peer review) is the author/funder, who has granted bioRxiv a license to display the preprint in perpetuity. It is made available under aCC-BY-NC-ND 4.0 International license.

\begin{tabular}{|c|c|c|c|c|c|c|c|c|c|c|c|c|}
\hline & \multicolumn{4}{|c|}{ iGluR } & mGluR & D2R & \multicolumn{2}{|c|}{ D1/5R } & \multicolumn{4}{|c|}{$\mathbf{G A B A}_{\mathbf{A}} R$} \\
\hline & dSPN & iSPN & ChI & FSI & ChI & ChI & ChI & FSI & dSPN & iSPN & ChI & FSI \\
\hline df & 8 & 7 & 7 & 7 & 3 & 6 & 6 & 7 & 8 & 8 & 8 & 7 \\
\hline $\mathbf{t}$ & 6.9 & 3.4 & 3.1 & 3.8 & 3.7 & 3.7 & 7.4 & 4.5 & 3.0 & 2.5 & 3.8 & 8.5 \\
\hline $\mathbf{p}$ & 0.0001 & 0.011 & 0.017 & 0.0063 & 0.034 & 0.0099 & 0.0003 & 0.0029 & 0.017 & 0.035 & 0.005 & 0.01 \\
\hline
\end{tabular}

Supplemental Table 1 Paired t-test for antagonist effects shown in Fig 2. 
bioRxiv preprint doi: https://doi.org/10.1101/2020.10.11.334961; this version posted October 11,2020 . The copyright holder for this preprint (which was not certified by peer review) is the author/funder, who has granted bioRxiv a license to display the preprint in perpetuity. It is made available under aCC-BY-NC-ND 4.0 International license.

\begin{tabular}{|c|c|c|c|c|c|c|c|c|c|c|c|c|}
\hline & \multicolumn{4}{|c|}{ iGluR } & mGluR & D2R & \multicolumn{2}{c|}{ D1/5R } & \multicolumn{4}{c|}{ GABA R } \\
\cline { 2 - 13 } & dSPN & iSPN & ChI & FSI & ChI & ChI & ChI & FSI & dSPN & iSPN & ChI & FSI \\
\hline psill & 900 & 220 & 720 & 430 & 80 & 12 & 120 & 0.25 & 51000 & 51000 & 70000 & 2600 \\
\hline nugget & 0 & 0 & 70 & 0 & 0 & 2 & 20 & 0 & 100 & 100 & 0 & 0 \\
\hline range & 0.25 & 0.25 & $\begin{array}{c}0.4 \\
0\end{array}$ & $\begin{array}{c}0.2 \\
5\end{array}$ & 0.40 & 0.30 & 0.40 & 0.30 & 0.30 & 0.25 & 0.35 & 0.20 \\
\hline
\end{tabular}

Supplemental Table 2 Experimental variogram fitting parameters. 


\begin{tabular}{|r|c|c|c|c|}
\cline { 2 - 5 } \multicolumn{1}{c|}{ dSPN } & Clust. 1 & Clust. 2 & Clust. 3 & F \\
\hline Location $^{1}$ & $\begin{array}{c}\text { Med. NAc } \\
\text { Ant.Lat.Dors. CPu }\end{array}$ & $\begin{array}{c}\text { Lat. NAc } \\
\text { Ant. CPu }\end{array}$ & Post. CPu & \\
& 721 & 2011 & 1445 & \\
\hline N of grid points & 42.3 & 13.7 & 16.1 & \multirow{2}{*}{4137} \\
\hline iGluR (pA) mean & 13.6 & 5.8 & 4.3 & \\
\hline SD & 208.3 & 194.6 & 106.9 & \multirow{2}{*}{647} \\
\hline GABA A $^{\text {(pA) mean }}$ & 83.9 & 93.8 & 45.4 & \\
\hline SD & & & & \\
\hline
\end{tabular}

\begin{tabular}{|c|c|c|c|c|}
\hline iSPN & Clust. 1 & Clust. 2 & Clust. 3 & $\mathbf{F}$ \\
\hline Location & $\begin{array}{c}\text { Med. NAc } \\
\text { Ant.Lat.Dors. CPu }\end{array}$ & Ant.Med. CPu & $\begin{array}{l}\text { Lat. NAc } \\
\text { Post. CPu }\end{array}$ & \\
\hline $\mathrm{N}$ of grid points & 672 & 958 & 2547 & \\
\hline $\begin{array}{r}\text { iGluR (pA) mean } \\
S D \\
\end{array}$ & $\begin{array}{c}17.9 \\
6.7 \\
\end{array}$ & $\begin{array}{l}7.2 \\
3.7 \\
\end{array}$ & $\begin{array}{l}8.4 \\
3.7 \\
\end{array}$ & 1459 \\
\hline $\begin{array}{r}\mathbf{G A B A}_{\mathbf{A}}(\mathbf{p A}) \text { mean } \\
S D\end{array}$ & $\begin{array}{c}135.8 \\
59.5\end{array}$ & $\begin{array}{c}213.6 \\
76.9\end{array}$ & $\begin{array}{l}87.9 \\
37.3\end{array}$ & 2001 \\
\hline
\end{tabular}

\begin{tabular}{|r|c|c|c|c|c|c|c|}
\cline { 2 - 8 } \multicolumn{1}{c|}{ ChI } & Clust. 1 & Clust. 2 & Clust. 3 & Clust. 4 & Clust. 5 & Clust. 6 & F \\
\hline Location & NAc & Ant. CPu & Med. CPu & Lat. CPu & Dors. CPu & Post. CPu & \\
\hline N of grid points & 394 & 1227 & 447 & 679 & 685 & 745 & \\
\hline iGluR (pA) mean & $\mathbf{6 5 . 8}$ & 17.5 & 19.2 & 11.7 & 12.9 & 12.4 & \multirow{2}{*}{1533} \\
SD & 25.1 & 12.3 & 8.9 & 5.2 & 4.3 & 4.3 & \\
\hline mGluR (pC) mean & 2.9 & 3.1 & 2.5 & $\mathbf{1 0 . 3}$ & 2.6 & 1.8 & \multirow{2}{*}{773} \\
SD & 0.8 & 1.6 & 0.7 & 6.8 & 1.3 & 0.7 & \\
\hline D2R (pC) mean & 3.0 & 4.1 & $\mathbf{7 . 4}$ & 3.4 & 4.8 & 3.5 & \multirow{2}{*}{119} \\
SD & 0.8 & 0.9 & 2.2 & 0.7 & 1.0 & 0.5 & \\
\hline D1/5R (pC) mean & 2.8 & 5.9 & 6.8 & $\mathbf{1 2 . 3}$ & 10.0 & 4.4 & \multirow{2}{*}{578} \\
SD & 0.6 & 3.6 & 3.4 & 5.3 & 5.0 & 2.1 & \\
\hline GABA (pA) mean & 142.1 & 284.1 & $\mathbf{3 8 4 . 2}$ & 187.7 & 163.5 & 122.4 & \multirow{2}{*}{1079} \\
SD & 47.3 & 79.7 & 93.5 & 77.8 & 55.8 & 72.9 & \\
\hline
\end{tabular}

\begin{tabular}{|c|c|c|c|c|c|c|}
\hline FSI & Clust. 1 & Clust. 2 & Clust. 3 & Clust. 4 & Clust. 5 & $\mathbf{F}$ \\
\hline Location & Vent. NAc & Ant. $\mathrm{CPu}$ & Ant.Med. CPu & $\begin{array}{c}\text { Med.Dors. } \mathrm{CPu} \\
\text { Ant.Dors.Lat } \mathrm{CPu}\end{array}$ & Post. CPu & \\
\hline $\mathrm{N}$ of grid points & 646 & 1628 & 303 & 229 & 1371 & \\
\hline $\begin{array}{r}\text { iGluR (pA) mean } \\
S D\end{array}$ & $\begin{array}{c}10.1 \\
3.7\end{array}$ & $\begin{array}{l}8.4 \\
2.9\end{array}$ & $\begin{array}{l}6.6 \\
2.3 \\
\end{array}$ & $\begin{array}{l}29.2 \\
10.4\end{array}$ & $\begin{array}{c}12.1 \\
4.5\end{array}$ & 1285 \\
\hline $\begin{array}{r}\mathrm{D} 1 / 5 \mathrm{R}(\mathrm{pC}) \text { mean } \\
S D\end{array}$ & $\begin{array}{r}0.26 \\
0.10 \\
\end{array}$ & $\begin{array}{l}0.50 \\
0.16\end{array}$ & $\begin{array}{l}0.98 \\
0.17\end{array}$ & $\begin{array}{l}0.41 \\
0.10 \\
\end{array}$ & $\begin{array}{l}0.28 \\
0.09\end{array}$ & 2180 \\
\hline $\begin{array}{r}\mathrm{GABA}_{\mathrm{A}}(\mathrm{pA}) \text { mean } \\
S D\end{array}$ & $\begin{array}{c}24.6 \\
5.1\end{array}$ & $\begin{array}{l}35.6 \\
12.8\end{array}$ & $\begin{array}{c}32.1 \\
6.9\end{array}$ & $\begin{array}{l}46.1 \\
10.6\end{array}$ & $\begin{array}{c}23.5 \\
5.9\end{array}$ & 524 \\
\hline
\end{tabular}

1. Location abbreviation: anterior (Ant), posterior (Post), medial (Med), lateral (Lat), ventral (Vent), dorsal (Dors).

2. Numbers in bold indicate maximum values for each response type, corresponding to the largest font size in Fig 4, right panel.

Supplemental Table 3 Descriptive statistics of cell-type clusters and anatomical locations 
bioRxiv preprint doi: https://doi.org/10.1101/2020.10.11.334961; this version posted October $11,2020$. The copyright holder for this preprint (which was not certified by peer review) is the author/funder, who has granted bioRxiv a license to display the preprint in perpetuity. It is made available under aCC-BY-NC-ND 4.0 International license.

\begin{tabular}{|c|c|c|c|c|c|}
\hline Glutamate & Clust. 1 & Clust. 2 & Clust. 3 & Clust. 4 & $\mathbf{F}$ \\
\hline Location & NAc & $\begin{array}{c}\text { Ant.Med. } \mathrm{CPu} \\
\text { Ant.Lat.Vent. CPu }\end{array}$ & Ant.Lat.Dors. $\mathrm{CPu}$ & Post. $\mathrm{CPu}$ & \\
\hline $\mathrm{N}$ of grid points & 417 & 1777 & 746 & 1237 & \\
\hline dSPN iGluR (pA) mean & $\begin{array}{l}40.1 \\
14.8\end{array}$ & $\begin{array}{c}12.5 \\
4.5\end{array}$ & $\begin{array}{l}30.9 \\
14.8\end{array}$ & $\begin{array}{c}15.7 \\
4.1\end{array}$ & 1697 \\
\hline $\begin{array}{r}\text { iSPN iGluR (pA) mean } \\
S D\end{array}$ & $\begin{array}{c}17.2 \\
5.4\end{array}$ & $\begin{array}{l}7.3 \\
4.7 \\
\end{array}$ & $\begin{array}{c}13.6 \\
6.4\end{array}$ & $\begin{array}{l}8.1 \\
1.9 \\
\end{array}$ & 780 \\
\hline $\begin{array}{r}\text { ChI iGluR (pA) mean } \\
S D \\
\end{array}$ & $\begin{array}{l}\mathbf{6 6 . 7} \\
23.5 \\
\end{array}$ & $\begin{array}{c}17.2 \\
9.3 \\
\end{array}$ & $\begin{array}{l}9.1 \\
3.5 \\
\end{array}$ & $\begin{array}{r}13.7 \\
4.7 \\
\end{array}$ & 3504 \\
\hline $\begin{array}{r}\text { FSI iGluR (pA) mean } \\
S D\end{array}$ & $\begin{array}{c}15.5 \\
9.4\end{array}$ & $\begin{array}{l}7.3 \\
2.5 \\
\end{array}$ & $\begin{array}{c}15.4 \\
9.1\end{array}$ & $\begin{array}{r}11.7 \\
3.3 \\
\end{array}$ & 578 \\
\hline ChI mGluR (pC) mean & $\begin{array}{l}3.0 \\
0.7\end{array}$ & $\begin{array}{l}2.9 \\
1.6\end{array}$ & $\begin{array}{l}9.5 \\
6.8\end{array}$ & $\begin{array}{l}2.1 \\
1.0\end{array}$ & 1006 \\
\hline
\end{tabular}

\begin{tabular}{|r|c|c|c|c|c|}
\cline { 2 - 6 } \multicolumn{1}{l|}{ Dopamine } & Clust. 1 & Clust. 2 & Clust. 3 & Clust. 4 & F \\
\hline Location & NAc & Ant.Med. CPu & Ant.Lat.Dors. CPu & Post. CPu & \\
\hline N of grid points & 904 & 938 & 1210 & 1125 & \\
\hline ChI D2R (pC) mean & 3.4 & $\mathbf{5 . 9}$ & 4.0 & 3.8 & \multirow{2}{*}{657} \\
SD & 0.8 & 2.2 & 1.1 & 0.8 & \\
\hline ChI D1/5R (pC) mean & 3.7 & 5.2 & $\mathbf{1 2 . 6}$ & 5.4 & \multirow{2}{*}{1533} \\
SD & 2.3 & 2.2 & 5.1 & 2.7 & \\
\hline FSI D1/5R (pC) mean & 0.28 & 0.72 & 0.38 & 0.34 & \multirow{2}{*}{1525} \\
SD & 0.09 & 0.23 & 0.17 & 0.11 & \\
\hline
\end{tabular}

\begin{tabular}{|c|c|c|c|c|c|}
\hline GABA & Clust. 1 & Clust. 2 & Clust. 3 & Clust. 4 & $\mathbf{F}$ \\
\hline Location & $\begin{array}{c}\text { NAc } \\
\text { Ant.Vent. CPu }\end{array}$ & Ant.Med.Dors. CPu & Ant.Lat.Dors. $\mathrm{CPu}$ & Post. $\mathrm{CPu}$ & \\
\hline $\mathbf{N}$ of grid points & 1177 & 701 & 858 & 1441 & \\
\hline $\begin{array}{r}\text { dSPN (pA) mean } \\
S D\end{array}$ & $\begin{array}{c}145.2 \\
58.8\end{array}$ & $\begin{array}{c}265.7 \\
98.9\end{array}$ & $\begin{array}{c}217.4 \\
75.1\end{array}$ & $\begin{array}{c}105.8 \\
45.4\end{array}$ & 1111 \\
\hline $\begin{array}{r}\text { iSPN (pA) mean } \\
S D\end{array}$ & $\begin{array}{c}106.2 \\
54.8\end{array}$ & $\begin{array}{c}188.7 \\
68.5\end{array}$ & $\begin{array}{c}177.1 \\
79.1\end{array}$ & $\begin{array}{l}76.8 \\
32.7\end{array}$ & 907 \\
\hline $\begin{array}{r}\text { ChI }(p A) \text { mean } \\
S D\end{array}$ & $\begin{array}{l}235.9 \\
103.0\end{array}$ & $\begin{array}{c}357.4 \\
89.0\end{array}$ & $\begin{array}{c}158.6 \\
63.8\end{array}$ & $\begin{array}{c}168.4 \\
83.8\end{array}$ & 904 \\
\hline $\begin{array}{r}\text { FSI (pC) mean } \\
S D\end{array}$ & $\begin{array}{l}32.1 \\
14.5\end{array}$ & $\begin{array}{c}30.9 \\
8.2\end{array}$ & $\begin{array}{l}37.1 \\
11.4\end{array}$ & $\begin{array}{c}24.4 \\
6.3\end{array}$ & 280 \\
\hline
\end{tabular}

Supplemental Table 4 Descriptive statistics of transmitter clusters and anatomical locations 